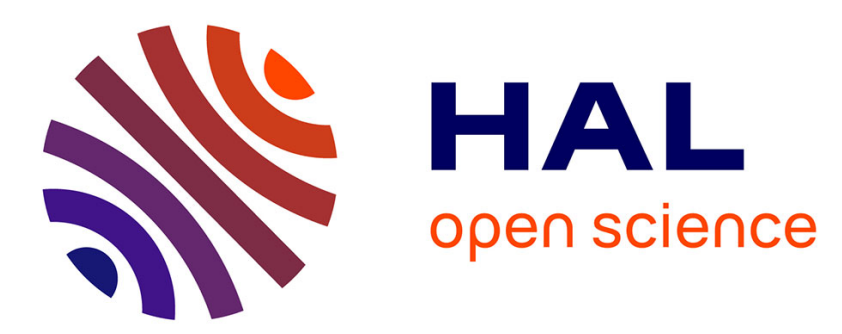

\title{
Uncertainty propagation and experimental verification of nonlinear viscoelastic sandwich beams
}

V Silva, Antonio Marco Gonçalves de Lima, Noureddine Bouhaddi, Helder Barbieri Lacerda

\section{- To cite this version:}

V Silva, Antonio Marco Gonçalves de Lima, Noureddine Bouhaddi, Helder Barbieri Lacerda. Uncertainty propagation and experimental verification of nonlinear viscoelastic sandwich beams. Mechanical Systems and Signal Processing, 2019, 132, pp.654 - 669. hal-03427061

\section{HAL Id: hal-03427061 \\ https://hal.science/hal-03427061}

Submitted on 12 Nov 2021

HAL is a multi-disciplinary open access archive for the deposit and dissemination of scientific research documents, whether they are published or not. The documents may come from teaching and research institutions in France or abroad, or from public or private research centers.
L'archive ouverte pluridisciplinaire HAL, est destinée au dépôt et à la diffusion de documents scientifiques de niveau recherche, publiés ou non, émanant des établissements d'enseignement et de recherche français ou étrangers, des laboratoires publics ou privés. 


\section{Uncertainties quantification and experimental verification of nonlinear viscoelastic sandwich beams}

\section{V.A.C. Silva ${ }^{a}$, A.M.G. de Lima ${ }^{a^{*}}$, N. Bouhaddi ${ }^{b}$, H.B. Lacerda ${ }^{a}$}

a Federal University of Uberlândia - School of Mechanical Engineering

Campus Santa Mônica - P.O. Box 593, CEP 38400-902 - Uberlândia, MG, Brazil

${ }^{\mathrm{b}}$ Univ. Bourgogne Franche-Comte, FEMTO-ST Institute, CNRS/UFC/ENSMM/UTBM

Department of Applied Mechanics, 2500, Besancon, France

The nonlinear dynamic analysis of viscoelastic systems is not an easy task. In some cases, it is due to the difficulty in dealing with the temperature- and frequency-dependent properties of the viscoelastic substructure. Also, since the real-life viscoelastic treatments are characterized by inherent uncertainties affecting their efficiency, their handling in the nonlinear modeling is essential from an engineering point of view. In this contribution, a stochastic modeling based on the Karhunen-Loève expansion is proposed for a three-layer sandwich beam having nonlinear behavior using the concept of complex modulus and shift factor. The nonlinear frequency responses were obtained by using an approximated harmonic balance method with the Galerkin bases. Due to the difficulty in solving the resulting complex nonlinear eigenproblem with a frequency-dependent stiffness, making the stochastic nonlinear analyses very costly, it is proposed an iterative reduction method to approximate the complex eigenmodes. The influence of the forcing amplitude and temperature on the computed nonlinear frequency responses has been confirmed by performing laboratory experiments with a three-layer sandwich beam specimen placed 
inside an environmental chamber. Also, a curve-fitting has been performed to calibrate the deterministic nonlinear model using optimization tools. The envelopes of nonlinear responses demonstrate the relevance of considering the uncertainties in design variables.

Keywords: Passive control, nonlinear vibrations, viscoelastic materials, stochastic finite element, parametric uncertainties.

\section{Introduction.}

In the open literature, a number of passive control techniques have been proposed to mitigate undesirable vibrations and so avoid catastrophes. Among those possibilities, constrained viscoelastic layers (CVLs) [1] are typically used in many applications such as automobiles, airplanes, communications satellites, bridges and building structures $[2$, 3]. However, the resolution of the equations of motion for structures with viscoelastic materials are relevant aspects to be considered, since their mechanical properties depends on frequency and operation temperature [4]. This is a reason for which linear vibrations analyses of viscoelastically-damped systems have been performed [5-7] for the purposes of vibration attenuation. However, due to the frequent occurrence of large displacements in most of the practical applications of CVLs, the vibrations induced by the geometric nonlinearities differ significantly from those of linear approaches [8], and a nonlinear modeling is found to be necessary.

For undamped nonlinear models, it is relatively common to use the asymptotic numerical method or the multimodal approach to perform nonlinear vibrations analyses [9-12]. However, for nonlinear structures containing viscoelastic materials, the nonlinear 
vibration analysis, especially in the frequency-domain, is not an easy task, owing to the supplementary difficulty in dealing with the frequency- and temperature-dependent behavior of the viscoelastic substructure.

Among the fundamental early studies regarding the nonlinear vibration analysis of sandwich structures, the work by Kovac et al. [13] is of great relevance. They have performed numerical and experimental studies with a three-layer sandwich beam having geometric nonlinearities by using a Kelvin-Voight model to account for the frequencydependent behavior of the damping. In the sequence, Hyer et al. [14] have developed approximate dynamic equations for nonlinear analyses of an axially restrained sandwich beam using the concept of hereditary integrals and the complex modulus. Iu et al. [15] have proposed the incremental harmonic balance method for the nonlinear analysis of multilayer sandwich beams containing soft viscoelastic cores. Xia and Lukaziewicz [16, 17] have addressed the nonlinear vibrations of sandwich plates by using a Kelvin-Voigt model for the viscoelastic core. Lee [18] have developed a finite element (FE) formulation combined with a viscoelastic material model for the nonlinear analysis in time-domain of sandwich beams under large displacements. Daya et al. [19] have conducted numerical studies with a sandwich beam having nonlinear behavior by means of the one-mode Galerkin method and the harmonic balance approach to obtain a frequency-amplitude relationship. Jacques et al. [8] have implemented a refined zig-zag model to deal with nonlinear vibrations of sandwich beams. The results have been compared with those obtained by Kovac et al. [13].

More recently, there have also been continued effort in the proposition of efficient FE modeling strategies of viscoelastic systems to handle both geometrical nonlinearities and/or viscoelasticity. As an example, Bilasse et al. [20] have used the harmonic balance 
method with the Galerkin bases to study the nonlinear vibrations of a three-layer sandwich beam incorporating a soft viscoelastic core. Mahmoudkhani and Haddadpour [21] have investigated the influence of narrow-band random excitations on the nonlinear responses of sandwich plates with incompressible viscoelastic cores. They have applied a fifth-order perturbation method in conjunction with the Galerkin bases in order to construct a phaseamplitude equation to be solved using a difference method.

In summary, it can be seen that, many researches on the nonlinear vibrations of viscoelastic sandwich structures have been conducted by some authors using the FE method in conjunction with the harmonic balance approach and Galerkin bases. However, since for more realistic applications, the parameters that control the efficiency of CVLs are characterized by inherent uncertainties [22-24], a natural extension of the nonlinear modeling capability is to account for these uncertainties, aiming at evaluating their influence on the nonlinear model predictions. Also, since it is too difficult to accurately model viscoelastic systems having geometrical nonlinearities, it is of great relevance to characterize them experimentally to calibrate the nonlinear FE model. Unfortunately, few works have dedicated to these subjects, which motivates the present study.

Hence, it is proposed a nonlinear stochastic viscoelastic FE model based on the Karhunen-Loève expansion [25] combined with a parameterization scheme to assess the nonlinear frequency responses variability. The Latin-Hyper-Cube (LHC) sampling [26] is used herein as stochastic solver. However, due to the high computational cost needed to compute the envelopes of stochastic nonlinear responses, owing to the large number of samples required for convergence, performed on the full nonlinear FE model, it motivates the use of an efficient iterative enriched reduction method based on the works by de Lima et al. [22], Cunha-Filho et al. [27] and Rouleau et al. [28]. 
Also, by placing a three-layer sandwich beam under large displacements inside an environmental chamber, the effects of the excitation-force amplitude and temperature of the viscoelastic material on the linear and nonlinear behaviors of the viscoelastic beam can be established. Furthermore, the measured and computed frequency responses of the nonlinear system are compared with the aim of calibrating the nonlinear FE model.

\section{Background on FE modeling of sandwich beams under large displacements}

This section summarizes the FE modeling of a moderately three-layer symmetric sandwich beam with geometrical nonlinearities based on the model proposed by Daya et al. [19] and implemented by Bilasse [20]. Figure 1 illustrates the element composed by a base-beam (1), a viscoelastic core (2) and a passive constraining layer (3).

\section{[Figure 1 about here]}

In the development of the nonlinear deterministic FE model accounting for the zig-zag effects [29], the Euler-Bernoulli's theory is assumed for the face layers and the Timoshenko's theory is adopted for the viscoelastic core. The constitutive materials are homogeneous and isotropic, but the viscoelastic part has a frequency- and temperaturedependent complex modulus, as discussed later in this section. The transverse bending displacement, $w(x, t)$, of the beam is the same for both layers and the shear strain in the viscoelastic core results from the difference between the in-plane displacements, $u_{i}(x, t)$

, of the face layers, $i=1,3$, by the relation, $u_{1,3}(x, t)=u(x, t) \pm\left[h_{v} \beta(x, t)-h_{e} \partial w(x, t) / \partial x\right] / 2$ 
, where $u_{2}(x, t)=u(x, t)$ and $\beta(x, t)$ are, respectively, the axial displacement and rotation of the middle-plane $\left(z_{2}=0\right)$ of the viscoelastic core.

Since, the sandwich beam is subjected to large amplitude deflections, the resulting geometrical nonlinearities effects can be introduced using the Von Karman's theory [19], resulting in the following nonlinear strain-displacement relations [20]:

$$
\begin{aligned}
& \varepsilon_{i}(x, z, t)=u_{i}^{\prime}(x, t)+\frac{1}{2} w^{\prime}(x, t)^{2}-\left(z-z_{i}\right) \beta_{i}^{\prime}(x, t) \quad i=1,2,3 \\
& \gamma_{2}(x, z, t)=\beta(x, t)+w^{\prime}(x, t)
\end{aligned}
$$

where $\beta_{1}(x, t)=\beta_{3}(x, t)=w^{\prime}(x, t), \beta_{2}(x, t)=\beta(x, t)$ and $\gamma_{2}(x, z, t)$ is the shear strain of the viscoelastic core, with $w^{\prime}=\partial w / \partial x$ and $\beta^{\prime}=\partial \beta / \partial x$.

By considering the Bernoulli and Timoshenko kinematical assumptions for the elastic and viscoelastic layers, respectively, the virtual work principle due to a transverse harmonic excitation, $F$, can be established, and the following axial equilibrium and flexural problems are constructed as [20]:

$$
\int_{0}^{L} N \delta u^{\prime} d x=0
$$

$$
\overbrace{\int_{0}^{L} N w^{\prime} \delta w^{\prime} d x}^{\text {Nonliner }}+\overbrace{\int_{0}^{L}\left[M_{\beta} \delta \beta^{\prime}+M_{w} \delta w^{\prime \prime}+T\left(\delta w^{\prime}+\delta \beta\right)+\left(2 \rho_{e} A_{e}+\rho_{v} A_{v}\right) \ddot{w} \delta w\right] d x}^{\text {Linear }}=\int_{0}^{L} F \delta w d x
$$


where $\delta u, \delta w$ and $\delta \beta$ are the components of the virtual displacement vector, $N$ and $T$ are the axial and shear forces, respectively, and $M_{w}$ and $M_{\beta}$ represent the bending moments. These expressions are given in details in reference [19].

The exact solution of Eq. (2) cannot be obtained easily and the FE method will be used retained herein. The element used in the discretization contains two nodes and three degrees of freedom (DOFs) per node: the transverse displacement, $w(x, t)=W(x) e^{i \omega t}$, the slope, $w^{\prime}(x, t)=W^{\prime}(x) e^{i \omega t}$, and the rotation, $\beta(x, t)=B(x) e^{i \omega t}$. The displacement amplitude, $W$, is approximated by using a cubic shape function and a linear interpolation function is assumed for the rotation, $B$, resulting in the following element vector:

$$
\left\{\begin{array}{l}
W \\
B
\end{array}\right\}=\left[\begin{array}{l}
\boldsymbol{N}_{w} \\
\boldsymbol{N}_{\beta}
\end{array}\right] \boldsymbol{u}^{(e)}
$$

where $N_{w}$ and $N_{\beta}$ represent the matrices formed by the interpolations functions, and $\boldsymbol{u}^{(e)}=\left[W_{i} W_{i}^{\prime} B_{i}\right]^{T}$, with $i=1$ to 2 , is the vector containing the nodal variables.

Upon using the above listed assumptions and Eqs. (1) and (3), following standard analytical developments based on variational approaches [30], the elementary matrices of the linear contribution in the flexural problem (2.b) can be derived as fallow [20]:

$$
\begin{aligned}
& \boldsymbol{M}^{(e)}=\left(2 \rho_{e} A_{e}+\rho_{v} A_{v}\right) \overline{\boldsymbol{M}}^{(e)} \\
& \boldsymbol{K}_{e}^{(e)}=\frac{E_{e} A_{e} h_{v}^{2}}{2} \overline{\boldsymbol{K}}_{e_{1}}^{(e)}-\frac{E_{e} A_{e} h_{e} h_{v}}{2} \overline{\boldsymbol{K}}_{e_{2}}^{(e)}+\left(2 E_{e} I_{e}+\frac{E_{e} A_{e} h_{e}^{2}}{2}\right) \overline{\boldsymbol{K}}_{e_{3}}^{(e)} \\
& \boldsymbol{K}_{v}^{(e)}(\omega, T)=2 I_{v}\left(1+v_{v}\right) G(\omega, T) \overline{\boldsymbol{K}}_{v_{1}}^{(e)}+A_{v} G(\omega, T) \overline{\boldsymbol{K}}_{v_{2}}^{(e)}
\end{aligned}
$$


where $\overline{\boldsymbol{M}}^{(e)}=\int_{x=0}^{L} \boldsymbol{N}_{w}^{T} \boldsymbol{N}_{w} d x, \overline{\boldsymbol{K}}_{e_{1}}^{(e)}=\overline{\boldsymbol{K}}_{v_{1}}^{(e)}=\int_{0}^{L} \boldsymbol{N}_{\beta}^{\prime T} \boldsymbol{N}_{\beta} d x, \overline{\boldsymbol{K}}_{e_{2}}^{(e)}=\int_{0}^{L}\left(\boldsymbol{N}_{\beta}^{\prime T} \boldsymbol{N}_{w}^{\prime \prime}+\boldsymbol{N}_{w}^{\prime \prime T} \boldsymbol{N}_{\beta}^{\prime}\right) d x$, $\overline{\boldsymbol{K}}_{e_{3}}^{(e)}=\int_{0}^{L} \boldsymbol{N}_{w}^{\prime \prime T} \boldsymbol{N}_{w}^{\prime \prime} d x$ and $\overline{\boldsymbol{K}}_{v_{2}}^{(e)}=\int_{0}^{L}\left(\boldsymbol{N}_{\beta}^{T} \boldsymbol{N}_{\beta}+\boldsymbol{N}_{\beta}^{T} \boldsymbol{N}_{w}^{\prime}+\boldsymbol{N}_{w}^{\prime T} \boldsymbol{N}_{\beta}+\boldsymbol{N}_{w}^{\prime T} \boldsymbol{N}_{w}^{\prime}\right) d x$ represent the parameterized elementary FE matrices.

In this study, the parameterization of the model, which is understood as a means of making those design parameters to appear explicitly in the elementary FE matrices, enables to account for the uncertainties in a straightforward way and facilitates the computation of the envelopes of nonlinear frequency responses. Thus, after assembling the elementary matrices to generate the global equations of motion associated with the free vibrations of the linear viscoelastic system with $N$ DOFs, it is possible to generate the following complex eigenproblem:

$$
\left[\boldsymbol{K}_{e}+G\left(\omega_{j}, T_{v}\right) \overline{\boldsymbol{K}}_{v}-\lambda_{j}^{*} \boldsymbol{M}\right] \boldsymbol{\phi}_{j}=\boldsymbol{O}
$$

where $\boldsymbol{K}_{e} \in R^{N \times N}$ is the stiffness matrix of the elastic faces, $\boldsymbol{M} \in R^{N \times N}$ is the mass matrix and $\overline{\boldsymbol{K}}_{v} \in R^{N \times N}$ is the frequency- and temperature-independent viscoelastic stiffness matrix for which the complex shear modulus, $G\left(\omega_{j}, T_{v}\right)$, has been factored-out. $\phi_{j}$ and $\lambda_{j}^{*}=\left(\omega_{j}^{*}\right)^{2}=\lambda_{j}^{\prime}+i \lambda_{j}^{\prime \prime}$, are the complex eigenvectors and eigenvalues, respectively, with $j=1$ to $N$. The circular frequency, $\omega_{j}$, and the modal loss factor, $\eta_{j}$, can be obtained by the relations, $\omega_{j}=\sqrt{\lambda^{\prime}}$ and $\eta_{j}=\lambda^{\prime \prime} / \lambda^{\prime}$. 
Clearly, the difficulty in solving the complex eigenproblem (5) comes from the fact that, the stiffness of the viscoelastic part is frequency- and temperature-dependent. As a result, it must be solved iteratively. However, when large-scale FE models and/or uncertainties are dealt with, such procedures involve costly computations, motivating the proposition of the inverse iteration algorithm shown in Fig. 2 to be used in conjunction with a model reduction method to compute the eigenpairs, according to the main steps:

Initialization: a basis, $\boldsymbol{T}=\left[\boldsymbol{\phi}^{0} \boldsymbol{R}_{e} \boldsymbol{R}_{v}\right]$, is constructed to perform the reduction of the FE matrices (4), where the initial basis, $\boldsymbol{\phi}^{0}=\left[\boldsymbol{\phi}^{0}, \quad, \boldsymbol{\phi}_{N R}^{0}\right]$, containing $N R$ modes is obtained by solving the eigenproblem (6) of the associated conservative viscoelastic system, and $\boldsymbol{R}=\boldsymbol{K}_{0}^{-1} \boldsymbol{F}$ and $\boldsymbol{R}_{v}=\boldsymbol{K}_{0}^{-1} \overline{\boldsymbol{K}}_{v} \phi^{0}$ are, respectively, the static residues due to the external excitations and viscoelastic forces, with $\boldsymbol{K}_{0}=\boldsymbol{K}_{e}+G_{0} \overline{\boldsymbol{K}}_{v}$.

$$
\left[\boldsymbol{K}_{0}-\left(\omega^{0}\right)^{2} \boldsymbol{M}\right] \boldsymbol{\phi}^{0}=\mathbf{0}
$$

The next steps, $k$, are performed until the relations, $\varepsilon_{1}>10^{-12}$ and $\varepsilon_{2}>10^{-3}$, are satisfied, where, at the beginning, $k=1$, it is assumed that, $\left(\omega_{1}, \boldsymbol{\phi}\right)=\left(\omega^{0}, \boldsymbol{\phi}^{0}\right)$.

Step 1: computation of the reduced dynamic stiffness matrix and its derivative with respect to frequency, $\omega_{k}$, given by:

$$
\begin{aligned}
& \boldsymbol{Z}_{k}\left(\omega_{k}, T_{v}\right)=\boldsymbol{T}^{T}\left[\boldsymbol{K}_{e}+G\left(\omega_{k}, T_{v}\right) \overline{\boldsymbol{K}}_{v}-\left(\omega_{k}\right)^{2} \boldsymbol{M}\right] \boldsymbol{T}^{T} \\
& \boldsymbol{Z}_{k}^{d}\left(\omega_{k}, T_{v}\right)=\frac{\partial \boldsymbol{Z}_{k}\left(\omega_{k}, T_{v}\right)}{\partial \omega_{k}}=\boldsymbol{T}^{T}\left[\frac{\partial G\left(\omega_{k}, T_{v}\right)}{\partial \omega_{k}} \overline{\boldsymbol{K}}_{v}-2 \omega_{k} \boldsymbol{M}\right] \boldsymbol{T}^{T}
\end{aligned}
$$


where $\partial G\left(\omega_{k}, T_{v}\right) / \partial \omega_{k}$ is the derivative of the complex modulus with respect to, $\omega_{k}$.

Step 2: solve the relation, $\boldsymbol{Z}_{k}\left(\omega_{k}, T_{v}\right) \boldsymbol{u}_{k}=\boldsymbol{Z}_{k}^{d}\left(\omega_{k}, T_{v}\right) \phi_{k}$, for, $\boldsymbol{u}_{k}$.

Step 3: compute the new eigenpair, $\omega_{k+1}=\omega_{k}+\frac{\boldsymbol{r} \phi_{k}}{\boldsymbol{r} \boldsymbol{u}_{k}}$ and $\boldsymbol{\phi}_{k+1}=\frac{\boldsymbol{u}_{k}}{\boldsymbol{r} \boldsymbol{u}_{k}}$;

Step 4: compute the errors, $\varepsilon_{1}=\left\|\boldsymbol{Z}_{k}\left(\omega_{k}, T_{v}\right) \boldsymbol{\phi}_{k}\right\|$ and $\varepsilon_{2}=\left|\left(\omega_{k+1}-\omega_{k}\right) / \omega_{k+1}\right|$.

[Figure 2 about here]

\subsection{Amplitude equation for the nonlinear FE model}

For the nonlinear FE model, it is assumed that, the response of the beam is periodic in time to a transverse loading of the form, $F(x, t)=f(x) e^{i \omega t}$, and parallel to a single linear vibration mode. However, the transverse displacement and rotation are dependent on the complex amplitude, $A$, given by, $w(x, t)=A W(x) e^{i \omega t}$ and $\beta(x, t)=A B(x) e^{i \omega t}$. The unknown, $A$, is determined by using the Galerkin approach, where $[W(x) B(x)]^{T}$ is the Galerkin's basis to be estimated using the results of the eigenproblem (5).

Since, the interest is to solve the flexural problem (2.b) to determine the complex stiffness accounting for the geometrical nonlinearities, it can be done by inserting the previous relations for $w(x, t)$ and $\beta(x, t)$ into the axial problem (2.a) and assuming that, the nonlinear terms in the strains relations (1) induce harmonics 0 and $2 \omega$, according to the von Karman's theory. It enables to determine the axial responses $u(x, t)$ and $N(x, t)$ 
as functions of the amplitude, $A$. After, by introducing these expressions into Eq. (2.b), with $\delta \beta(x)=B(x) e^{-i \omega t}$ and $\delta w(x)=W(x) e^{-i \omega t}$, one gets the amplitude equation:

$$
\left[k_{e}+G\left(\omega, T_{v}\right) \bar{k}_{v}-\omega^{2} m\right] A+k_{n l}\left(\omega, T_{v}\right) \bar{A} A^{2}=f
$$

In Eq. (8), the linear modal parameters are computed using the model described in Section 2 by the relations, $m=\boldsymbol{\phi}^{T} \boldsymbol{M} \boldsymbol{\phi}, k_{e}=\boldsymbol{\phi}^{T} \boldsymbol{K}_{e} \boldsymbol{\phi}, \bar{k}_{v}=\boldsymbol{\phi}^{T} \overline{\boldsymbol{K}}_{v} \boldsymbol{\phi}$ and $f=\boldsymbol{\phi}^{T} \boldsymbol{F}$, where $\phi=T \phi$ and $\phi$ are the eigenvectors of the reduced system obtained by the method shown in Fig. 2. $k_{n l}\left(\omega, T_{v}\right)=k_{n l}^{0}+G\left(2 \omega, T_{v}\right) \bar{k}_{n l}^{1}$ and the frequency- and temperatureindependent nonlinear modal stiffnesses are given as:

$$
\begin{aligned}
& k_{n l}^{0}=\left[\frac{E_{e} A_{e}+G_{0}\left(1+v_{v}\right) A_{v}}{L / 2}\right]\left(\boldsymbol{\phi}^{T} \overline{\boldsymbol{K}}_{n l} \boldsymbol{\phi}\right)^{2}+\frac{E_{e} A_{e}}{L}\left|\boldsymbol{\phi}^{T} \overline{\boldsymbol{K}}_{n l} \boldsymbol{\phi}\right|^{2} \\
& \bar{k}_{n l}^{1}=\left(A_{v} / L\right)\left(1+v_{v}\right)\left|\boldsymbol{\phi}^{T} \overline{\boldsymbol{K}}_{n l} \boldsymbol{\phi}\right|^{2}
\end{aligned}
$$

where $\overline{\boldsymbol{K}}_{n l}={ }_{e=1}^{\text {nelem }} \overline{\boldsymbol{K}}_{n l}^{(e)}$, with $\overline{\boldsymbol{K}}_{n l}^{(e)}=\int_{0}^{L} \boldsymbol{N}_{w}^{\prime T} \boldsymbol{N}_{w}^{\prime} d x$, symbol $\bigcup$ indicates matrix assembling and $G_{0}$ is the value of the complex modulus in the low frequency range [22].

Since the stiffnesses coefficients appearing in Eq. (8) are frequency-dependent, to solve it for a given temperature, $T_{v}$, in the vicinity of any circular frequency, $\omega$, these coefficients are writing in a complex form, as suggested by Bilasse et al. [20]. Thus, after some mathematical manipulations, it leads to the generalized equation (10), which can be solved, for instance, by using the solve function available in MATLAB ${ }^{\circledR}$. 


$$
f_{3}\left(\omega, T_{v}\right) r^{3}+f_{2}\left(\omega, T_{v}\right) r^{2}+f_{1}\left(\omega, T_{v}\right) r^{1}+f_{0}=0
$$

where $r=A^{2}, f_{0}=-|f|^{2}, f_{3}\left(\omega, T_{v}\right)=\left(k_{n l}^{0}+G^{\prime}\left(2 \omega, T_{v}\right) \bar{k}_{n l}^{1}\right)^{2}+\left(G^{\prime \prime}\left(2 \omega, T_{v}\right) \bar{k}_{n l}^{1}\right)^{2}$,

$f_{2}\left(\omega, T_{v}\right)=2\left(k_{n l}^{0}+G^{\prime}\left(2 \omega, T_{v}\right) \bar{k}_{n l}^{1}\right)\left(k_{e}+G^{\prime}\left(\omega, T_{v}\right) \bar{k}_{v}-\omega^{2} m\right)+\ldots$

$$
\ldots 2 G^{\prime \prime}\left(\omega, T_{v}\right) \bar{k}_{v} G^{\prime \prime}\left(2 \omega, T_{v}\right) \bar{k}_{n l}^{1}
$$

$f_{1}\left(\omega, T_{v}\right)=\left(k_{e}+G^{\prime}\left(\omega, T_{v}\right) \bar{k}_{v}-\omega^{2} m\right)^{2}+\left(G^{\prime \prime}\left(\omega, T_{v}\right) \bar{k}_{v}\right)^{2} . G^{\prime}(\omega)$ and $G^{\prime \prime}(\omega)$ represent the storage modulus and loss modulus of the viscoelastic material, respectively.

\section{Stochastic nonlinear FE model.}

In the quest for the stochastic modeling, in practical applications of CVLs, the uncertainties are primarily those associated to the thicknesses of the layers and to the temperature of the viscoelastic part [15]. Thus, the choice of these variables as uncertain in this study is an important aspect to be considered. Within this aim, the Karhunen-Loève (KL) expansion is used to represent the random fields [25]. For example, the stochastic elementary mass matrix is computed as, $\overline{\boldsymbol{M}}^{(e)}(\theta)=\int_{x=0}^{L} H(x, \theta) \boldsymbol{N}_{w}^{T} \boldsymbol{N}_{w} d x$, where the random field, $H(x, \theta)$, can be expressed by its mean, $\bar{H}(x)=\varepsilon[H(x, \theta)]$, and its covariance, $C\left(x_{1}, x_{2}\right)=\varepsilon\left\{\left[H\left(x_{1}, \theta\right)-\bar{H}\left(x_{1}\right)\right]\left[H\left(x_{2}, \theta\right)-\bar{H}\left(x_{2}\right)\right]\right\}$, where $\theta$ is the random process, $x$, is the spatial dependence of the random field and $\varepsilon(\bullet)$ is the expectation operator. 
If, $H(x, \theta)$, is a homogeneous Gaussian random process with a symmetric and positive-definite covariance defined in a physical domain, $\Omega$, it is possible to find a unique projection of it on an orthonormal truncated random basis as follows [25]:

$$
H(x, \theta)=\bar{H}(x)+\sum_{r=1}^{n} \sqrt{\lambda_{r}} f_{r}(x) \xi_{r}(\theta)
$$

where $f_{r}(x)$ and $\lambda_{r}$ are the eigenfunctions and eigenvalues of the covariance.

Hence, for the sandwich beam element shown in Fig. 1, the analytical solution to the eigenproblem proposed by Ghanem and Spanos [25] and implemented by Guedri et al. [23] for the $\mathrm{KL}$ into the elementary FE domain $\Omega_{x}=[-L / 2, L / 2]$, is given as:

$$
\lambda_{r}=\frac{2 c^{2}}{\omega_{r}^{2}+c^{2}}, \quad f_{r}(x)=\alpha_{r} g_{r}(x)
$$

In Eq. (12), for $r(r \geq 1)$ odd, $\alpha_{r}=1 / \sqrt{L+\sin \left(2 \omega_{r} L\right) / 2 \omega_{r}}, g_{r}(x)=\cos \left(\omega_{r} x\right)$ and $\omega_{r}$ is the solution of the transcendental function, $c-\omega_{r} \tan \left(\omega_{r} L\right)=0$, defined into the domain, $\left[(r-1) \frac{\pi}{L},\left(r-\frac{1}{2}\right) \frac{\pi}{L}\right]$. However, for $r(r \geq 2)$ even, $g_{r}(x)=\sin \left(\omega_{r} x\right)$, $\alpha_{r}=1 / \sqrt{L-\sin \left(2 \omega_{r} L\right) / 2 \omega_{r}}$, and $\omega_{r}$ is the solution of, $\omega_{r}+c \tan \left(\omega_{r} L\right)=0$, defined into the domain, $\left[\left(r-\frac{1}{2}\right) \frac{\pi}{L}, r \frac{\pi}{L}\right] \cdot c=1 / L$ is the correlation length.

Thus, based on the KL expansion detailed previously, the stochastic elementary FE matrices of the linear sandwich viscoelastic beam are found as follow: 


$$
\begin{aligned}
& \boldsymbol{M}^{(e)}(\theta)=\boldsymbol{M}^{(e)}+\sum_{r=1}^{n} \overline{\boldsymbol{M}}_{r}^{(e)} \xi_{r}(\theta) \\
& \boldsymbol{K}_{e}^{(e)}(\theta)=\boldsymbol{K}_{e}^{(e)}+\sum_{r=1}^{n} \overline{\boldsymbol{K}}_{e_{r}}^{(e)} \xi_{r}(\theta) \\
& \boldsymbol{K}_{v}^{(e)}(\omega, T, \theta)=G(\omega, T) \overline{\boldsymbol{K}}_{v}^{(e)}+G(\omega, T, \theta) \sum_{r=1}^{n} \overline{\boldsymbol{K}}_{v_{r}}^{(e)} \xi_{r}(\theta)
\end{aligned}
$$

where $\boldsymbol{M}^{(e)}, \boldsymbol{K}_{e}^{(e)}$ and $\overline{\boldsymbol{K}}_{v}^{(e)}$ are defined in Eqs. (4), and $\overline{\boldsymbol{M}}_{r}^{(e)}=\int_{x=0}^{L} \sqrt{\lambda_{r}} f_{r}(x) \boldsymbol{N}^{T} \boldsymbol{N} d x$, $\overline{\boldsymbol{K}}_{e_{r}}^{(e)}=\int_{x=0}^{L} \sqrt{\lambda_{r}} f_{r}(x) \boldsymbol{D}_{k}^{T} \boldsymbol{C}_{k} \boldsymbol{D}_{k} d x, \overline{\boldsymbol{K}}_{v_{r}}^{(e)}=\int_{x=0}^{L} \sqrt{\lambda_{r}} f_{r}(x) \boldsymbol{D}_{v}^{T} \overline{\boldsymbol{C}}_{v} \boldsymbol{D}_{v} d x$ are the random matrices. Hence, after assembling the stochastic FE matrices, the random modal parameters can be obtained by the relations, $m(\theta)=\boldsymbol{\phi}(\theta)^{T} \boldsymbol{M} \phi(\theta), k_{e}(\theta)=\phi(\theta)^{T} \boldsymbol{K}_{e}(\theta) \phi(\theta)$, $k_{v}(\theta)=\boldsymbol{\phi}(\theta)^{T} \overline{\boldsymbol{K}}_{v}(\theta) \boldsymbol{\phi}(\theta), k_{n l}^{0}(\theta)=k_{n l}^{0}+\sum_{r=1}^{n} k_{n l_{r}}^{0} \xi_{r}(\theta)$ and $\bar{k}_{n l}^{1}(\theta)=\bar{k}_{n l}^{1}+\sum_{r=1}^{n} \bar{k}_{n l}^{1} \xi_{r}(\theta)$, where $\phi(\theta)$ are the random eigenvectors of the eigenproblem (5) at each sample, $\theta$. In the same way, the random nonlinear coefficients, $k_{n l_{r}}^{0}$ and $\bar{k}_{n l_{r}}^{1}$, can be computed by introducing the relation, $\overline{\boldsymbol{K}}_{n l_{r}}^{(e)}=\int_{0}^{L} \sqrt{\lambda_{r}} f_{r}(x) \boldsymbol{N}_{w}^{\prime T} \boldsymbol{N}_{w}^{\prime} d x$, in Eq. (9).

Hence, the random amplitude equation to be solved for the stochastic nonlinear viscoelastic beam subjected to a deterministic excitation can be expressed as:

$$
\left[k_{e}(\theta)+G\left(\omega, T_{v}, \theta\right) \bar{k}_{v}(\theta)-\omega^{2} m(\theta)\right] A(\theta)+k_{n l}(\theta) \bar{A}(\theta) A(\theta)^{2}=f
$$


where $k_{n l}\left(\omega, T_{v}, \theta\right)=k_{n l}^{0}(\theta)+G\left(2 \omega, T_{v}, \theta\right) \bar{k}_{n l}^{1}(\theta)$.

The thicknesses of the layers have been considered as uncertain parameters using the KL expansion based on the stochastic FE method. However, since the temperature does not appear explicitly in the FE matrices, but in the complex modulus, $G\left(\omega, T_{v}\right)$, the related uncertainties are introduced by the relation, $T_{v}(\theta)=T_{v}^{0}\left(1+\delta_{T} \xi(\theta)\right)$, where $\xi(\theta)$ is a Gaussian random variable and $T_{v}^{0}$ and $\delta_{T}$ are, respectively, the mean value of the temperature and its dispersion level. Also, for the purposes of the present study, the stochastic problem (14) is solved using the LHC sampling method [31].

\section{Numerical applications with the deterministic nonlinear FE model}

An academic example formed by a clamped-free three-layer sandwich beam has been used herein to highlight the main features and capabilities of the deterministic FE formulation. The model is composed by 100 finite elements to ensure the convergence of the FE method, where the base-beam and constraining layer are made of steel (Young's modulus, $E=1.93 \times 10^{11} \mathrm{~N} / \mathrm{m}^{2}$, mass density, $\rho=7900 \mathrm{~kg} / \mathrm{m}^{3}$, Poisson ratio, $v=0.3$ ), with a core of $3 \mathrm{M}$ ISD1 $12^{\mathrm{TM}}$ material [32] $\left(\rho=950 \mathrm{~kg} / \mathrm{m}^{3}, v=0.49\right)$, whose properties are given by Eq. (15) for frequency and temperature intervals of, $1 \mathrm{~Hz} \leq \omega \leq 10^{6} \mathrm{~Hz}$ and $210 K \leq T_{v} \leq 360 K$ [27]. The geometrical characteristics of the mean model are given below: the beam has a length of, $180 \mathrm{~mm}$, a width of, $33 \mathrm{~mm}$, and thicknesses of the elastic and viscoelastic layers of, $h_{e}=1 \mathrm{~mm}$ and $h_{v}=1 \mathrm{~mm}$, respectively. 


$$
\mathrm{G}\left(\omega, T_{v}\right)=0.4307+\frac{1200}{1+3.24 \times\left(\frac{i \omega_{r}}{1543000}\right)^{-0.18}+\left(\frac{i \omega_{r}}{1543000}\right)^{-0.6847}}[M P a]
$$

where $\alpha\left(T_{v}\right)=10^{\left(-3758.4 \times\left(\frac{1}{T_{v}}-0.00345\right)-225.06 \times \log \left(0.00345 \times T_{v}\right)+0.23273 \times\left(T_{v}-290\right)\right)}$ is the shift factor function and $\omega_{r}=\alpha\left(T_{v}\right) \omega$ is the so-called reduced frequency [4].

\subsection{Influence of the excitation-force amplitude on the frequency response.}

This first application is intended to evaluate the influence of the excitation-force amplitude on the nonlinear responses of the beam by solving the amplitude equation (9) for the nonlinear deterministic FE model. The computations consisted in plotting the amplitudes, $A$, corresponding to a transverse displacement, $w$, at the tip of the beam due to a transverse loading applied at the same point. The nominal value of the temperature of the viscoelastic material is adopted as, $30^{\circ} \mathrm{C}$.

Figure 3 shows the amplitudes of vibrations corresponding to the first flexural mode of the beam for different forcing amplitudes. It is interesting to observe that, as the amplitude of the excitation increases, the vibration becomes larger, showing the nonlinear characteristics of the beam for these loading conditions. Clearly, such nonlinearities can have strong influence on the stiffness and damping properties of the viscoelastic system, which can be verified by comparing the linear and nonlinear frequency response curves corresponding to the forcing amplitudes of, $f_{0}=0.1 \mathrm{~N}$ and $f_{0}=1 \mathrm{~N}$, respectively. 


\subsection{Influence of the complex eigenmodes on the frequency response.}

Another aspect of great relevance to be discussed here is the complex eigenmodes used in the computation of the nonlinear frequency responses of the viscoelastic beam. In this study, the complex eigenmodes have been obtained by solving Eq. (5) for a reducedorder FE model, according the method depicted in Fig. 2. Figure 4 shows that, at, $f_{0}=1 \mathrm{~N}$ , the imaginary parts of the complex eigenmodes, which are frequently disregarded in some eigenvalue approaches for constant viscoelastic laws [20], are essential to account for the whole temperature- and frequency-dependent viscoelastic damping effects. Since, the nonlinear vibrations are underestimated by taking into account only the real parts of the complex eigenmodes for the viscoelastic frequency-dependent law used herein.

\section{[Figure 4 about here]}

Also, it is demonstrated the effectiveness of the proposed reduction basis, $\boldsymbol{T}$, in approximating the nonlinear frequency responses of the beam. Figure 5 enables to compare the nonlinear responses computed for the full and reduced-order models using the following bases: $\boldsymbol{T}_{1}=\left[\begin{array}{ll}\boldsymbol{\phi}_{0} & \boldsymbol{R}\end{array}\right]$ (10 eigenmodes of CAS, $\boldsymbol{\phi}_{0}, 1$ static residual due to the external excitation, $\boldsymbol{R}$ ), and $\boldsymbol{T}_{2}=\left[\begin{array}{lll}\boldsymbol{\phi}_{0} & \boldsymbol{R} & \boldsymbol{R}_{v}\end{array}\right]$ (10 eigenmodes of CAS, $\boldsymbol{\phi}_{0}, 1$ static residual due to the external excitation, $\boldsymbol{R}$, and 8 static residuals due to the viscoelastic damping forces, $\boldsymbol{R}_{v}$, after SVD filtering). It is evident that, the first-order static residuals associated to the viscoelastic damping forces are essential to give reasonable accurate 
nonlinear frequency response approximations, as demonstrated by the agreement between the frequency responses amplitudes of the full and reduced-order FE models for, $\boldsymbol{T}_{2}$.

\section{[Figure 5 about here]}

In the quest for efficiency, Fig. 6 shows that, as the number of elements in the FEmesh increases, the reduction method provides a significantly computational gain of approximately $90 \%$ in the computations of the approximated nonlinear responses. Thus, it motivates its use to deal with large-scale nonlinear models or stochastic problems, as addressed later in this work, where the simulations using the full stochastic nonlinear FE model can be unfeasible, even for a simple academic example.

\section{[Figure 6 about here]}

\section{Updating the deterministic nonlinear FE model using experiments.}

Figure 7(b) reveals that, the correlation between the measured and computed responses of the beam displaying nonlinear behavior for, $f_{0}=4 N$, is not satisfactory. Clearly, it is difficult to accurately model higher order nonlinear behaviors, even if the FE model predicts, with reasonable accuracy, the frequency and amplitude of resonance of the beam displaying linear behavior, as shown in Fig. 7(a). In this case, the differences observed in the regions outside the resonance frequency may be associated with the difficulty in achieving a perfect mechanical boundary condition of the cantilever beam, 
contributing to an increasing in its damping capability, even if the it is mounted in a heavy rigid fixture, as shown in Fig. 8.

Hence, before considering the uncertainties through the stochastic nonlinear FE model, firstly, it is interesting to perform a curve-fitting to update the nonlinear stiffness experimentally to correctly capture the viscoelastic beam's dynamics. Also, it is verified experimentally the influence of the forcing amplitude and temperature of the viscoelastic material on the frequency responses of the beam.

\section{[Figure 7 about here]}

The experimental results shown in Fig. 7 for the linear and nonlinear behaviours have been measured using the experimental setup depicted in Fig. 8(a). The tests consisted in using the stepped-sine approach [33], to measure the frequency responses of the beam for various values of forcing amplitude and operation temperature. The strategy consists in increasing/decreasing the excitation frequency step-by-step, while the amplitude of the excitation and operation temperature are kept constant for all the forcing frequencies. To control the operation temperature, the sandwich beam specimen has been mounted inside an environmental chamber, model 877-GO-ESPEC, as indicated in Figs. 8(a) and 8(b). Then, the displacement of the system at each excitation frequency is acquired after the transient responses disappear and the frequency response curves can be constructed for the specific excitation-force amplitude in a frequency band of interest.

In all tests, the beam tip is subjected to a harmonic excitation through the Shaker, model TMS ${ }^{\circledR}$ K2007E01, connected to the free end of the cantilever sandwich beam by a stinger (see Fig. 8(a)). A load cell is placed between the beam tip and the stinger to 
monitor the input loading to the beam and the acceleration responses are measured with a piezoelectric accelerometer, model $352 \mathrm{C} 22 \mathrm{PCB}^{\circledR}$, placed at the same point. For data acquisition and processing, a LabView ${ }^{\circledR}$ code was developed to generate the excitation signal and to acquire the acceleration responses through National Instrument systems $\mathrm{NI}^{\circledR}$ 9269 and $\mathrm{NI}^{\circledR} 9233$, respectively. The code waits, at least, 10 seconds for the response to reach its steady-state prior to recording/exporting it to another MATLAB code to compute the amplitude versus frequency. Also, a feedback control has been implemented with the aim of monitoring/controlling the excitation force amplitude during the whole experiment to avoid disturbances.

\section{[Figure 8 about here]}

The interest is to adjust the nonlinear stiffness of the beam model until it has the same resonance peak as the experimental measure depicted in Fig. 7(b). Within this aim, it is used the following relation, $k_{n l}^{a d}\left(\omega, T_{v}\right)=c k_{n l}\left(\omega, T_{v}\right)$, where the unknown variable, $c$ , has been identified by using the well-known Differential Evolution (DE) algorithm [34] to solve the following unconstrained objective function:

$$
f_{o b j}=\sum_{j=1}^{n p}\left(A^{\mathrm{e}}\left(\omega_{j}\right)-A^{n}\left(\omega_{j}\right)\right)^{2}
$$

where $A^{\mathrm{e}}\left(\omega_{j}\right)$ and $A^{n}\left(\omega_{j}\right)$ are, respectively, the measured and computed amplitudes at frequency, $\omega_{j}$, and $n p$ is the number of points considered in the frequency bandwidth 
of, $20-50 \mathrm{~Hz}$, which comprises the first flexural vibration mode of the sandwich beam with a frequency resolution of $0.01 \mathrm{~Hz}$.

In the optimization problem, the admissible variation of the continuous parameter

is of, $c \in[0-1]$, since the resonance amplitude of the beam displaying nonlinear behavior is overestimated by the model (see Fig. 7(b)). Only this range is considered as lateral constraints, where the control variables of the DE algorithm are: number of generations, 50; population size: 50; crossover probability, 0.9; and weighted factor, 0.8 .

Figure 9 compares the nonlinear responses predicted by the adjusted model using the optimal value of, $c=1.57 \times 10^{-3}$, and the measured frequency response curves for various values of forcing amplitudes. In all tests, the temperature was kept constant at, $30^{\circ} \mathrm{C}$, by using the chamber. Now, the experimental measures are approximated quite accurately by the numerical predictions, even when the beam displays nonlinear behavior at higher values of forcing amplitude, since the shape of the resonance peak changes strongly from, $f_{0}=1 N$, to, $f_{0}=4 N$. Also, the measured frequency responses confirm the numerical results that, at higher values of excitation-force amplitudes, the viscoelastic beam displays nonlinear behaviors for a fixed temperature.

[Figure 9 about here]

Since, the temperature can be controlled by using the environmental chamber, it is also important to evaluate its degree of influence on the beam displaying nonlinear behavior at, $f_{0}=4 N$. It can be done by varying the temperature of the environmental chamber from $10^{\circ} \mathrm{C}$ to $35^{\circ} \mathrm{C}$. By examining Fig. 10 , one can conclude that, the agreement between the frequency responses predicted by the adjusted model and those obtained by 
measurements is considered very satisfactory. Also, it reveals that, as the temperature of the viscoelastic part increases, the resonance peak increases accordingly. It is expected, since for the $3 \mathrm{M}$ ISD112 ${ }^{\mathrm{TM}}$ material, the temperature range of interest is characterized by having a loss factor that decreases rapidly with increasing temperature, as shown in Fig. 11, at fixed resonance frequency value of, $35 \mathrm{~Hz}$.

In particular, for the temperature range between $10^{\circ} \mathrm{C}$ and $25^{\circ} \mathrm{C}$, the beam displays linear behaviors, even for the forcing amplitude of, $f_{0}=4 \mathrm{~N}$. But, for temperature values between $30^{\circ} \mathrm{C}$ to $35^{\circ} \mathrm{C}$, the nonlinear characteristics become clearly more pronounced, as indicated by the changes on the resonance peaks. In this temperature range, the hardening nonlinearity affects significantly the properties of the viscoelastic beam, resulting in an augmentation of its resonance frequency. It can be better visualized by examining Fig. 12, which compares the measured and computed resonance characteristics as functions of temperature. It is evident that, as the temperature increases, the resonance amplitude increases, and the resonance frequency decreases rapidly until it reaches its minimum value at $25^{\circ} \mathrm{C}$ due to the reduction of the loss factor of the viscoelastic material, as shown in Fig. 11. After this point, an increase in the temperature leads to an increase in the resonance frequency due to the hardening nonlinearity and an increase in the resonance amplitude due to the loss of efficiency of the viscoelastic material.

[Figure 10 about here]

[Figure 11 about here]

[Figure 12 about here] 


\section{Influence of the uncertainties on the response of the stochastic model.}

Now, the interest is to evaluate the degree of influence of the uncertainties on the frequency responses variabilities of the adjusted nonlinear stochastic FE model by solving the random amplitude equation (14) for several LHC realizations. The computations of the envelopes of frequency responses are performed by introducing the dispersion levels of $5 \%$ and $15 \%$ on the thicknesses of the layers and on the temperature of the viscoelastic system for a forcing amplitude of, $f_{0}=3 N$, according to the cases defined in Table 1 , based on the hypothesis that, all the random variables exhibit normal distributions with zero mean and unit variance [26].

Firstly, the number of LHC samples, $n_{s}$, required to assure the convergence of the nonlinear frequency responses variabilities must be identified. In this case, the meansquare error [24], $\operatorname{conv}\left(n_{s}\right)=\left(1 / n_{s}\right) \sum_{j=1}^{n_{s}}\left\|A_{j}(\theta)-A\right\|^{2}$, was taken into consideration here, where, $A$, is the amplitude of the adjusted mean FE model obtained in the previous section for, $f_{0}=4 N$, and, $A_{j}(\theta)$, is the random amplitude computed for a LHC sample, $j$. Figure 13 shows the evolutions of the mean-square error as a function of the number of samples for each uncertain parameter. It is seen that, the solutions always converge for, $n_{s}=400$, for each uncertain parameter. Thus, this number will be used in all stochastic simulations that follow to assure the minimum nonlinear frequency responses dispersion.

[Table 1 about here]

[Figure 13 about here] 
Figures 14 to 17 show the envelopes of random nonlinear frequency responses for a confidence level of $95 \%$ for the uncertainties affecting the design variables. It implies that, the nonlinear response is inside the minimum and maximum curves of the envelopes with a probability of, $95 \%$. It can be noted that, as the uncertainty levels increase, the response variability increases accordingly, especially for uncertainties introduced on the thickness of the elastic faces and on the temperature of the viscoelastic material. However, the uncertainties on the thickness of the elastic faces have the greatest influence on the resonance frequency, while the uncertainties concentrated on the operation temperature of the viscoelastic material exert a strongly influence on the resonance peak. Clearly, the confidence region becomes larger when the sources of uncertainties are concentrated in all the design variables simultaneously.

Hence, it helps the designer to evaluate the degree of influence of uncertainties on the frequency responses of a nonlinear viscoelastic system and its robustness with respect to random design parameters. However, the larger influence of the dispersions on the nonlinear responses shown in Fig. 17 reveals that, it is also important to know where the sources of uncertainties are concentrated in the system to improve its predictability for more realistic situations.

Finally, it is worth mentioning that, the numerical efficiency in the computations of the nonlinear frequency responses variabilities shown in Figs. 14 to 17 is due to the reduced-order model implemented herein, according to Fig. 2. Also, the parameterization of the model defined by Eq. (4) strongly facilitates to perform uncertainty propagations without re-assembling the elementary matrices of the mean FE model required to compute the linear and nonlinear modal parameters defined in Eq. (14). 
[Figure 14 about here]

[Figure 15 about here]

[Figure 16 about here]

[Figure 17 about here]

\section{Concluding remarks.}

In this paper, a stochastic modeling methodology has been proposed to explore the nonlinear behavior of viscoelastic beams subjected to large displacements, accounting for the dependence of the linear and nonlinear stiffnesses with respect to frequency and temperature. Firstly, to improve the accuracy of the deterministic nonlinear FE model in predicting the nonlinear responses of the viscoelastic beam, it has been performed a model updating for the nonlinear part using optimization tools by comparing the measured and computed nonlinear frequency responses. In the sequence, to demonstrate experimentally the influence of the forcing amplitude and temperature of the viscoelastic material on the behavior of the sandwich beam, experiments were conducted inside an environmental chamber using the stepped-sine approach. Finally, the adjusted stochastic nonlinear FE model was achieved by concentrating uncertainties on the thicknesses of the layers and on the temperature of the viscoelastic material to verify their influence on the shape bent resonance peaks. However, due to the high computational cost needed to compute the envelopes of nonlinear curves and to improve the numerical efficiency in the stochastic analysis, an adequate parameterization of the FE matrices combined with a reduced-order model were also addressed herein. 
The numerical and experimental results reveal that, for a given temperature of the viscoelastic beam having linear behavior, as the forcing amplitude increases, keeping the temperature constant, the beam displays nonlinear behavior over a range of forcing amplitude. It is seen that, the bent resonance peaks become more pronounced at high values of forcing amplitudes. However, for the situation in which the nonlinear operation condition is kept constant and the temperature of the viscoelastic material is decreased, keeping now the forcing amplitude constant, the behavior of the beam becomes linear. Thus, beyond the fact that the viscoelastic material has a significantly influence on the linear and nonlinear behaviors of the viscoelastic sandwich beam, the results enables to conclude about the effectiveness of the viscoelastic material in controlling nonlinear vibrations, even for higher values of forcing amplitudes.

The parameterization combined with the reduced-order model, as suggested in this study, has proved to be an adequate strategy to compute the modal parameters required to construct the nonlinear frequency responses of the beam subjected to uncertainties. The numerical applications revealed that, the uncertainties concentrated especially on the thickness of the elastic layers have the greatest influence on the resonance frequency and the temperature of the viscoelastic material has a greatest influence on the resonance peak of the nonlinear viscoelastic sandwich beam.

Hence, the envelopes of random nonlinear responses convey valuable information about the degree of influence of the uncertainties on design variables characterizing the viscoelastic sandwich beam under large displacements. However, it is of great relevance to know where the sources of uncertainties are concentrated in the nonlinear model in order to improve its predictability for more realistic situations. 


\section{Concluding remarks.}

The authors are grateful to CNPq for the continued support to their research activities, especially through the research grant 302026/2016-9 (A.M.G. de Lima). It is important to express the acknowledgements to the FAPEMIG.

\section{References.}

[1] E.M. Austin, Variations on modeling of constrained-layer damping treatments, Shock and Vibration Digest. 31 (1999) 275-280.

[2] M.D. Rao, Recent applications of viscoelastic damping for noise control in automobiles and commercial airplanes, in: Proceedings of the Emerging Trends in Vibration and Noise Engineering Symposium, Columbus, USA, 2001.

[3] B. Samali, K.C.S. Kwok, Use of viscoelastic dampers in reducing wind and earthquake induced motion of building structures, Engineering Structures. 17 (1995) 639654.

[4] A.D. Nashif, D.I.G. Jones, J.P. Henderson, Vibration damping, John Wiley \& Sons, New York, USA, 1985.

[5] A.G.C. Filho, A.M.G. de Lima, M.V. Donadon, L.S. Leão, Flutter suppresion of plates using passive constrained viscoelastic layers, Mechanical Systems and Signal Processing. 79 (2016) 99-111.

[6] E.A. Ribeiro, J.T. Pereira, C.A. Bavastri, Passive vibration control in rotor dynamics: Optimization of composed support using viscoelastic materials, Journal of Sound and Vibration. 351 (2015) 43-56. 
[7] B. Kovâcs, Vibration analysis of a damped arch using an iterative laminate model, Journal of Sound and Vibration. 254 (2002) 367-378.

[8] N. Jacques, E.M. Daya, M. Potier-Ferry, Nonlinear vibration of viscoelastic sandwich beams by the harmonic balance and finite element methods, Journal of Sound and Vibration. 329 (2010) 4251-4265.

[9] L. Azrar, E. Boutyour, M. Potier-Ferry, Nonlinear forced vibrations of plates by an asymptotic-numerical method, Journal of Sound and Vibration. 252 (2002) 657-674.

[10] R. Arquier, S. Bellizzi, R. Bouc, B. Cochelin, Two methods for the computation of nonlinear modes of vibrating systems at large amplitudes, Computers and Structures. 84 (2006) 1565-1576.

[11] A. Przekop, M.S. Azzouz, X. Guo, C. Mei, L. Azrar, Finite element multimode approach to nonlinear free vibrations of shallow shells, American Institute of Aeronautics and Astronautics Journal. 42 (2004) 2373-2381.

[12] Y. Gerges, E. Sadoulet-Reboul, M. Ouisse, N. Bouhaddi, Extension of modal reduction methods to non-linear coupled structure-acoustic problems, European Journal of Computational Mechanics. 20 (2011) 227-245.

[13] E.J. Kovac, W.J. Anderson, R.A. Scott, Forced non-linear vibrations of a damped sandwich beam, Journal of Sound and Vibration. 17 (1971) 25-39.

[14] M.W. Hyer, W.J. Anderson, R.A. Scott, Non-linear vibrations of three-layer beams with viscoelastic cores I. Theory, Journal of Sound and Vibration. 46 (1976) 121-136. [15] V.P. Iu, Y.K. Cheung, S.L. Lau, Non-linear vibration analysis of multilayer beams by incremental finite elements Part I: Theory and numerical formulation, Journal of Sound and Vibration. 100 (1985) 359-372. 
[16] Z.O. Xia, S. Lukasiewicz, Non-linear, free, damped vibrations of sandwich plates, Journal of Sound and Vibration. 175 (1994) 219-232.

[17] Z.O. Xia, S. Lukasiewicz, Non-linear damped vibrations of simply-supported rectangular sandwich plates, Nonlinear Dynamics. 8 (1995) 417-433.

[18] H.H. Lee, Non-linear vibration of a multilayer sandwich beam with viscoelastic layers, Journal of Sound and Vibration. 216 (1998) 601-621.

[19] E.M. Daya, L. Azrar, M. Potier-Ferry, An amplitude equation for the non-linear vibration of viscoelastically damped sandwich beams, Journal of Sound and Vibration. $271(2004) 789-813$

[20] M. Bilasse, E.M. Daya, L. Azrar, Linear and nonlinear vibrations analysis of viscoelastic sandwich beams, Journal of Sound and Vibration. 329 (2010) 4950-4969.

[21] S. Mahmoudkhani, H. Haddadpour, Nonlinear vibration of viscoelastic sandwich plates under narrow-band random excitations, Nonlinear Dynamics. 74 (2013) 165-188.

[22] A.M.G. de Lima, D.A. Rade, N. Bouhaddi, Stochastic modeling of surface viscoelastic treatments combined with model condensation procedures, Shock and Vibration. 17 (2010) 429-444.

[23] M. Guedri, A.M.G. de Lima, N. Bouhaddi, D.A. Rade, Robust design of viscoelastic structures based on stochastic finite element models, Journal of Mechanical Systems and Signal Processing. 24 (2010) 59-77.

[24] R. Sampaio, C. Soize, On measures of nonlinearity effects for uncertain dynamical systems applications to a vibro-impact system, Journal of Sound and Vibration. 303 (2007) 659-674.

[25] R.G. Ghanem, P.D. Spanos, Stochastic finite elements: A spectral approach, Spring Verlag, 1991. 
[26] B.V. Nieuwenhof, J.P. Coyette, Modal approaches for the stochastic finite element analysis of structures with material and geometric uncertainties, Computer Methods in Applied Mechanics and Engineering. 192 (2003) 3705-3729.

[27] A.G. Cunha-Filho, Y.P.J. Briend, A.M.G. de Lima, M.V. Donadon, An efficient iterative model reduction method for aeroviscoelastic panel flutter analysis in the supersonic regime, Mechanical Systems and Signal Processing. 104 (2018) 575-588.

[28] L. Rouleau, J.F. Deü, A. Legay, A comparison of model reduction techniques based on modal projection for structures with frequency-dependent damping, Mechanical Systems and Signal Processing. 90 (2017) 110-125.

[29] D.K. Rao, Frequency and loss factors of sandwich beams under various boundary conditions, Mechanical Engineering Science. 20 (1978) 271-282.

[30] O.C. Zienkiewicz, R.L. Taylor, The finite element method: Solid mechanics, Vol. 2, Butterworth-Heinemann, 2000.

[31] A. Florian, An efficient sampling scheme: updates Latin Hypercube sampling, Probabilistic Engineering Mechanics. 7 (1992) 123-130.

[32] Information on http://www.3m.com (Accessed July 2015)

[33] A. Carrella, D. J. Ewins, Identifying and quantifying structural nonlinearities in engineering applications from measured frequency response functions, Mechanical Systems and Signal Processing. 25 (2011) 1011-1027.

[34] L.F.F. Rodovalho, R.A. Borges, A.M.G. de Lima, T.N. Costa, Hybrid approach of optimization applied to an inverse problem in the dynamic modeling of a three-floor structure, Applied Soft Computing. 65 (2018) 412-427. 


\section{FIGURE CAPTIONS}

Figure 1 - Illustration of the three-layer sandwich beam element.

Figure 2 - Inverse iteration scheme used to predict the complex eigensolutions.

Figure 3 - Frequency responses of the nonlinear sandwich beam for various values of forcing amplitudes.

Figure 4 - Comparison between the frequency responses of the sandwich beam displaying nonlinear behavior at, $f_{0}=1 N$, accounting for the real and complex eigenmodes.

Figure 5 - Comparison between the frequency responses of the full and reduced-order models of the sandwich beam displaying nonlinear behavior at, $f_{0}=1 \mathrm{~N}$.

Figure 6 - Computational gain with the reduced model as a function of the mesh size.

Figure 7 - Comparison between the measured and computed linear (a) and nonlinear (b) frequency responses of the sandwich beam.

Figure 8 - (a) Schematic of the experimental nonlinear sandwich beam setup; (b) photography of the experimental setup.

Figure 9 -Comparison between the measured and computed frequency response curves of the nonlinear sandwich beam for various values of forcing amplitude.

Figure 10 - Comparison between the measured (a) and computed (b) frequency responses of the nonlinear sandwich beam for various values of operation temperature. Figure 11 - Variation of the loss factor of the 3M ISD112 with temperature at, $35 \mathrm{~Hz}$. Figure 12 - Comparison between the measured and computed resonance frequencies and resonance amplitudes of the nonlinear sandwich beam as functions of temperature. 
Figure 13 - Convergence of the mean-square error for the uncertainties concentrated on the thicknesses of the layers and on the temperature of the viscoelastic material.

Figure 14 - Envelopes of random frequency responses of the sandwich beam displaying nonlinear behavior at, $f_{0}=3 \mathrm{~N}$ and $T_{v}=30^{\circ} \mathrm{C}$, for uncertainty levels of $5 \%$ (left) and $15 \%$ (right) concentrated on the thickness of the viscoelastic core.

Figure 15 - Envelopes of random frequency responses of the sandwich beam displaying nonlinear behavior at, $f_{0}=3 N$ and $T_{v}=30^{\circ} \mathrm{C}$, for uncertainty levels of $5 \%$ (left) and $15 \%$ (right) concentrated on the thickness of the face layers.

Figure 16 - Envelopes of random frequency responses of the sandwich beam displaying nonlinear behavior at, $f_{0}=3 \mathrm{~N}$ and $T_{v}=30^{\circ} \mathrm{C}$, for uncertainty levels of $5 \%$ (left) and $15 \%$ (right) concentrated on the temperature of the viscoelastic material.

Figure 17 - Envelopes of random frequency responses of the sandwich beam displaying nonlinear behavior at, $f_{0}=3 \mathrm{~N}$ and $T_{v}=30^{\circ} \mathrm{C}$, for uncertainty levels of $5 \%$ (left) and $15 \%$ (right) concentrated in all design variables.

\section{TABLE CAPTIONS}

Table 1 - Uncertainty levels of the nonlinear viscoelastic sandwich beam. 


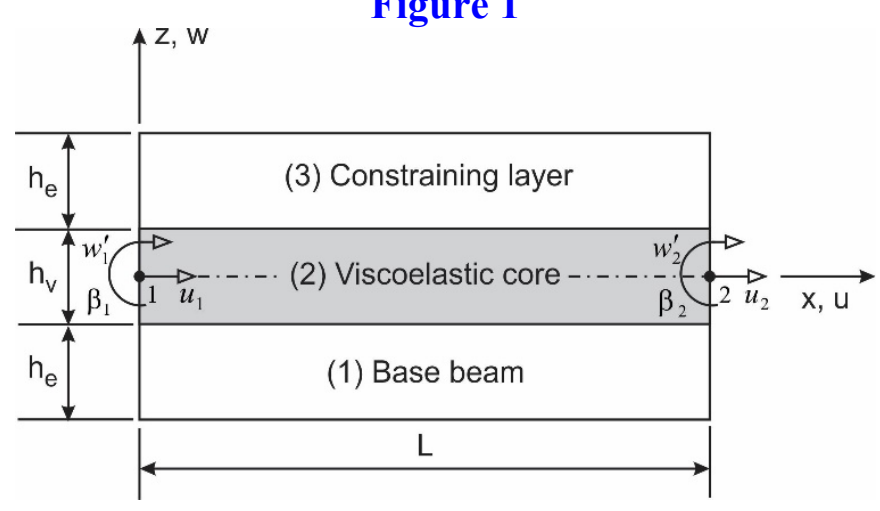

Figure 2

Algorithm: Inverse Iteration Method

Require: Initial eigenpair, $\left(\omega_{1}, \phi_{1}\right)=\left(\omega^{0}, \phi^{0}\right), r \phi_{0}=1$ and $k=1$

1. While $\varepsilon_{1}>10^{-12}$ and $\varepsilon_{2}>10^{-3}$ do

2. Solve, $\boldsymbol{Z}_{k}\left(\omega_{k}, T_{v}\right) \boldsymbol{u}_{k}=\boldsymbol{Z}_{k}^{d}\left(\omega_{k}, T_{v}\right) \boldsymbol{\phi}_{k}$, for, $\boldsymbol{u}_{k}$

3. Compute, $\omega_{k+1}=\omega_{k}+(\boldsymbol{r} \phi) /\left(\boldsymbol{r} \boldsymbol{u}_{k}\right)$

4. Normalize, $\boldsymbol{\phi}_{k+1}=\boldsymbol{u}_{k} /\left(\boldsymbol{r} \boldsymbol{u}_{k}\right)$

5. Compute, $\varepsilon_{1}=\left\|\boldsymbol{Z}_{k}\left(\omega_{k}, T_{v}\right) \phi_{k}\right\|$ and $\varepsilon_{2}=\left|\left(\omega_{k+1}-\omega_{k}\right) / \omega_{k+1}\right|$ 6. End while

Figure 3

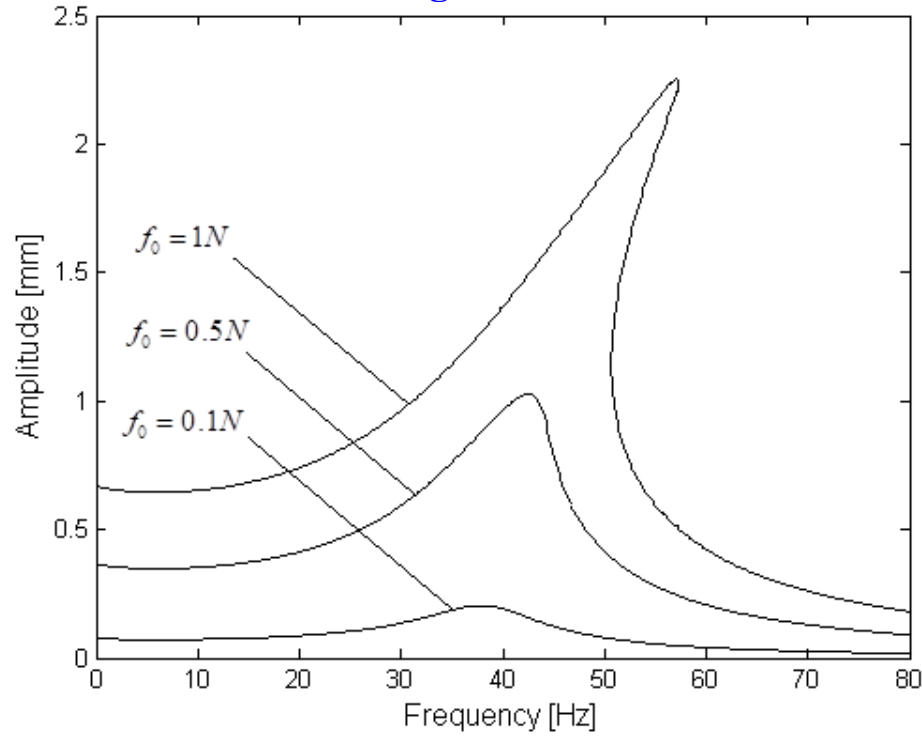


Figure 4

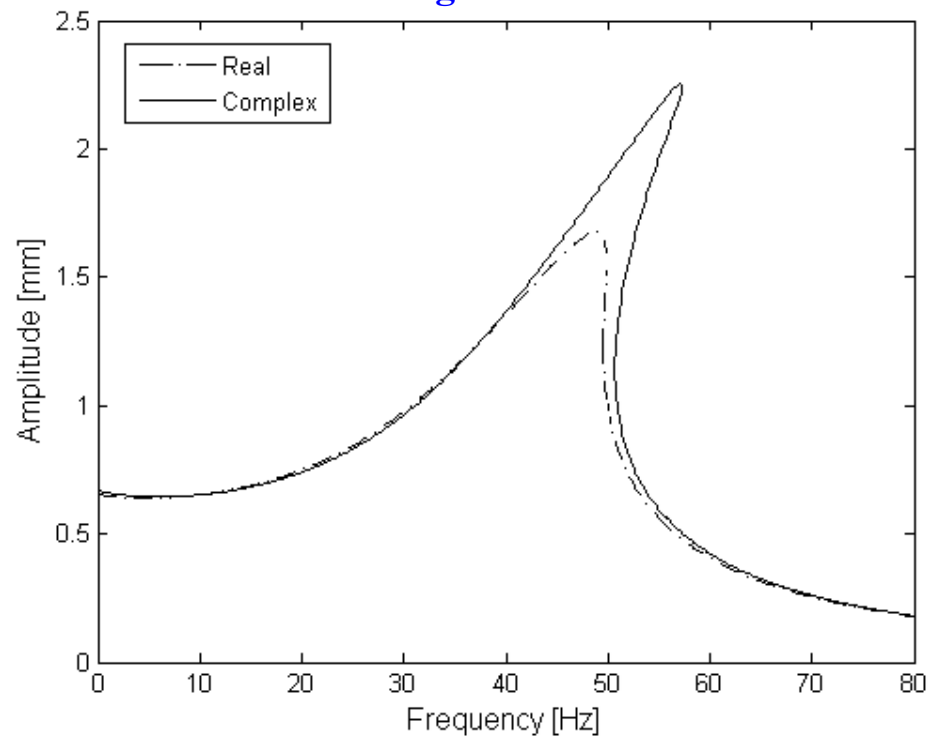

Figure 5
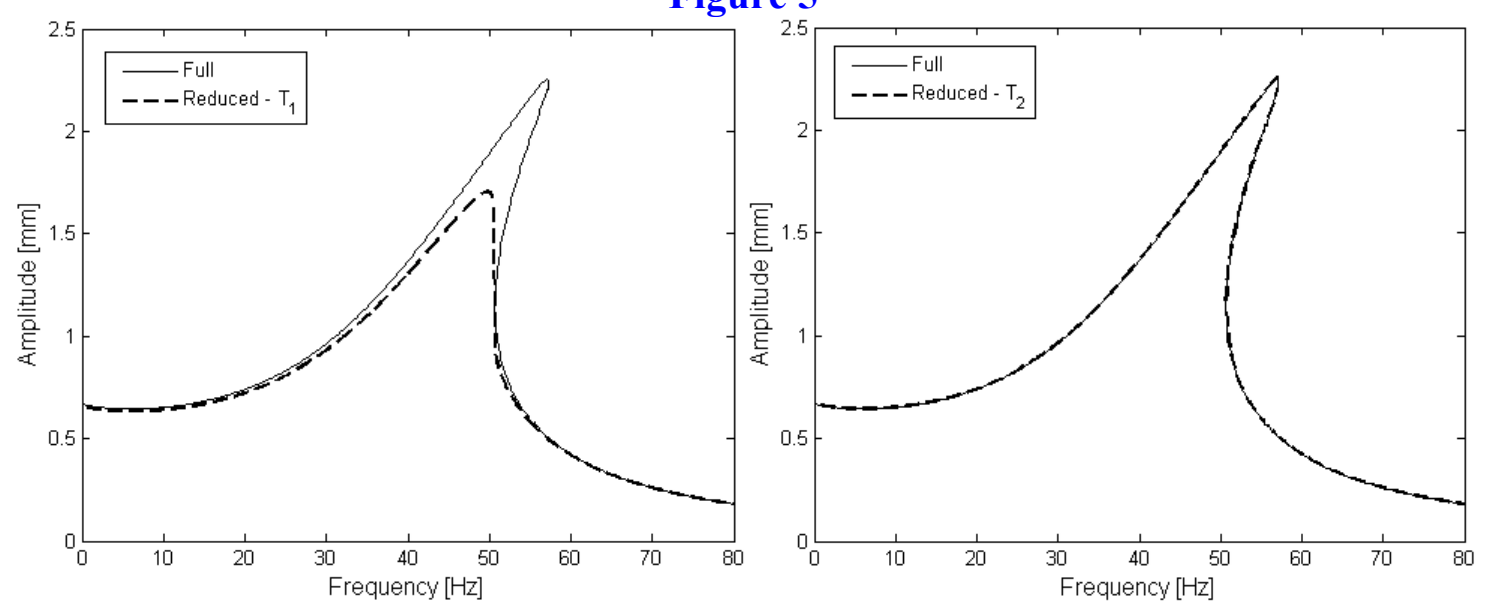

Figure 6

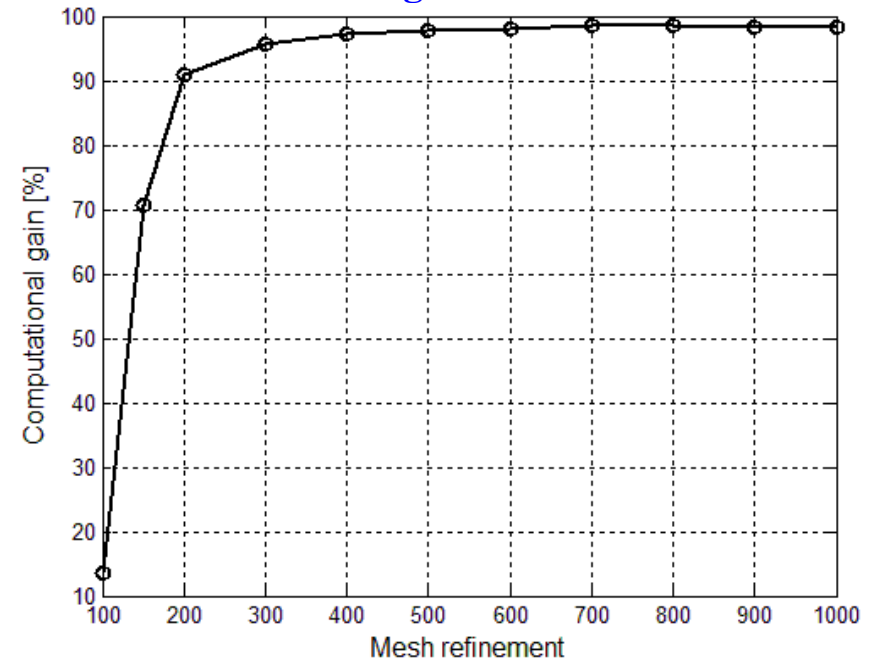


Figure 7

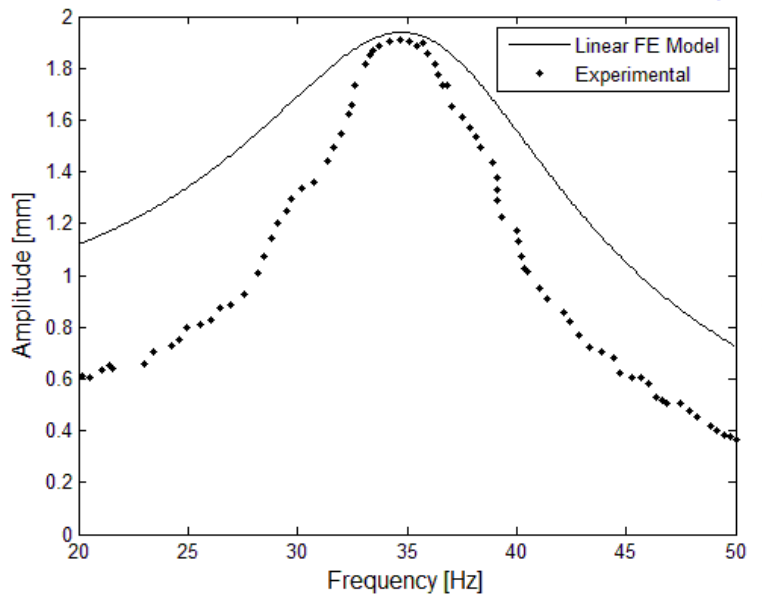

(a)

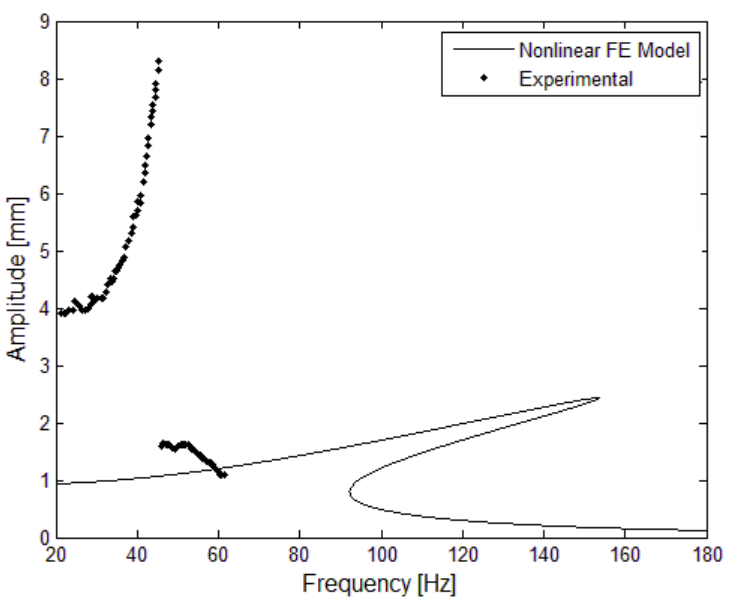

(b)

Figure 8

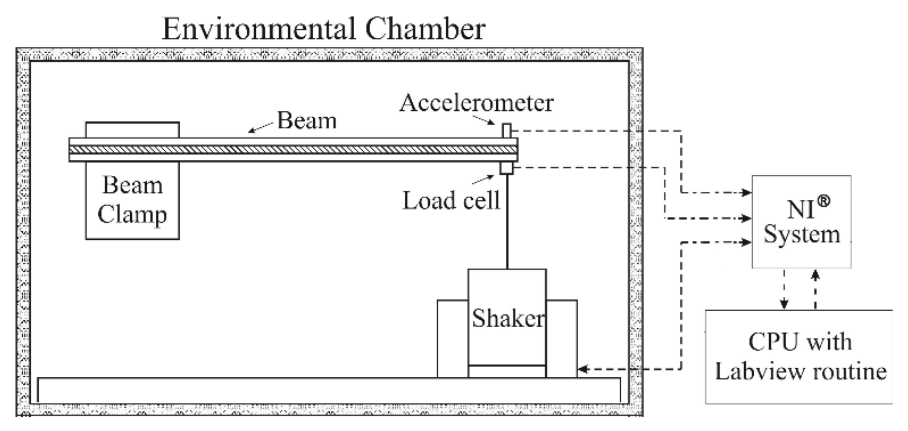

(a)

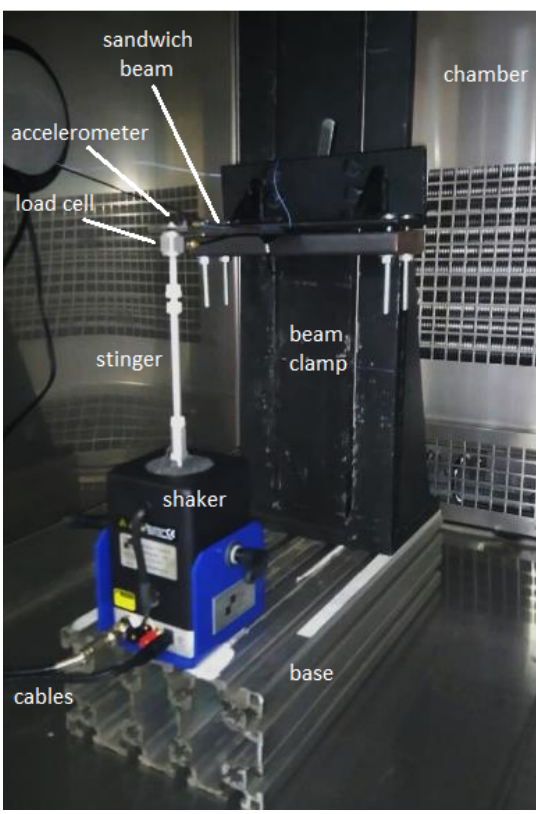

(b)

Figure 9
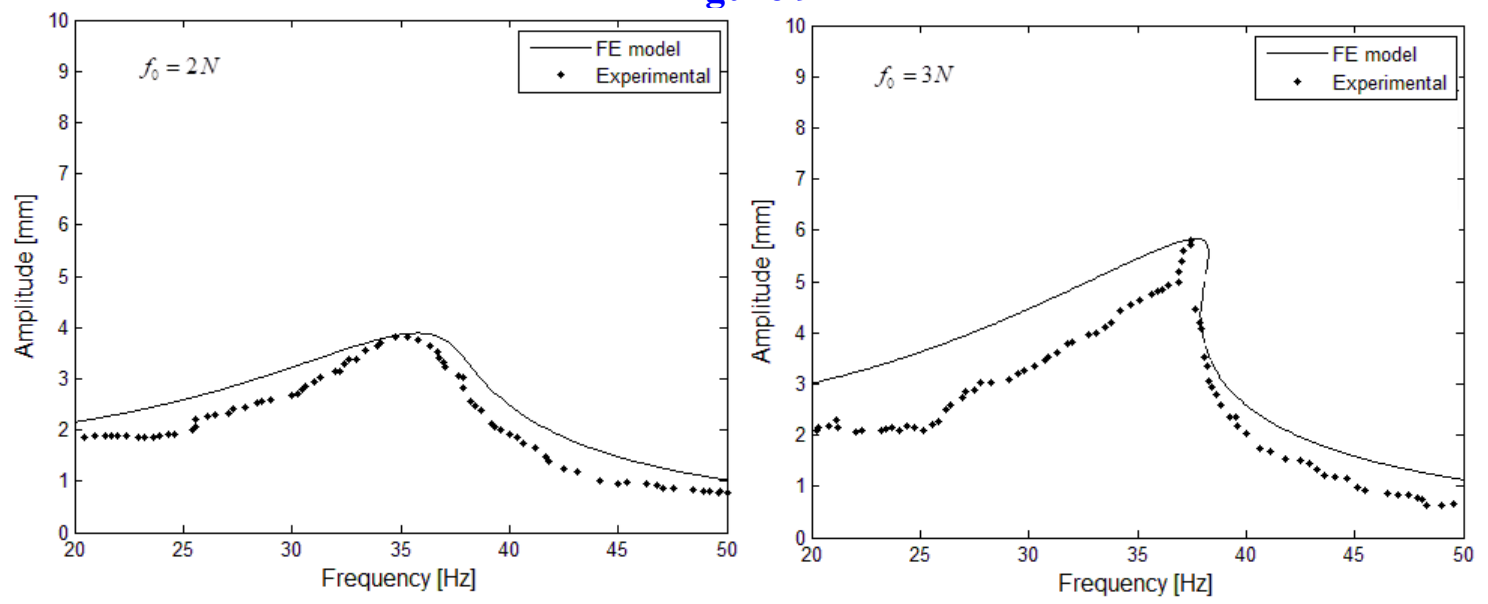

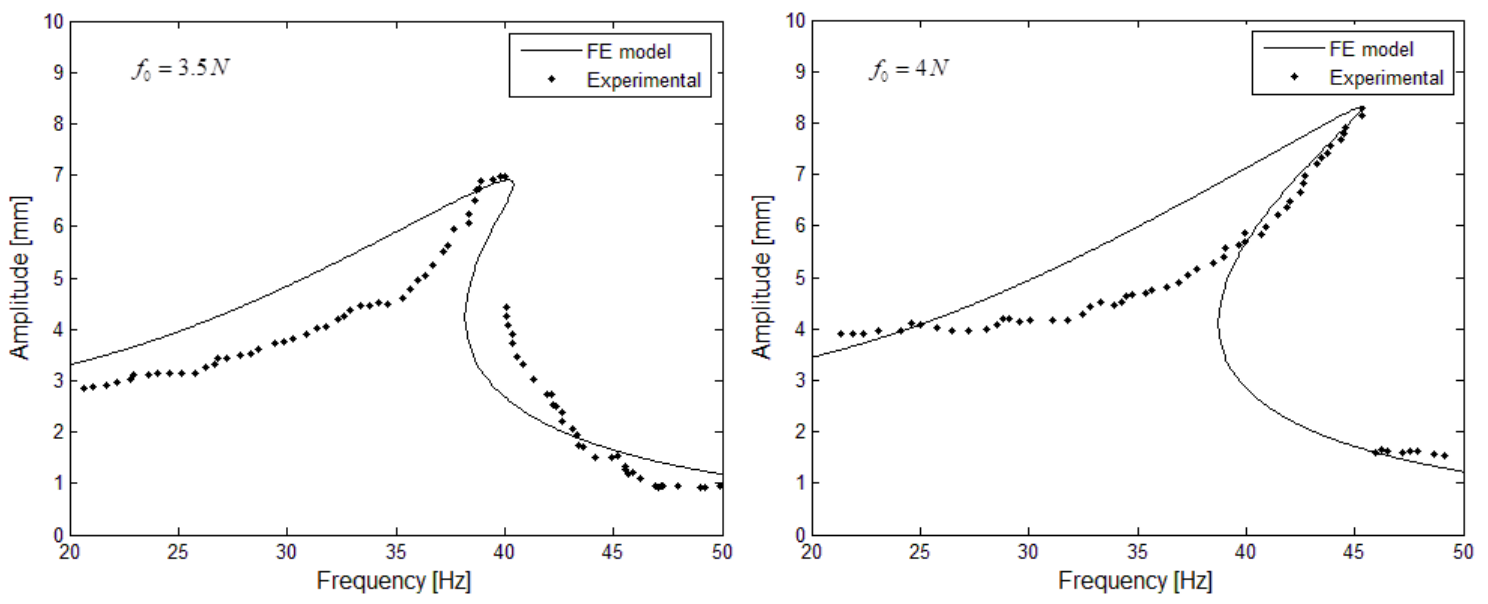

Figure 10

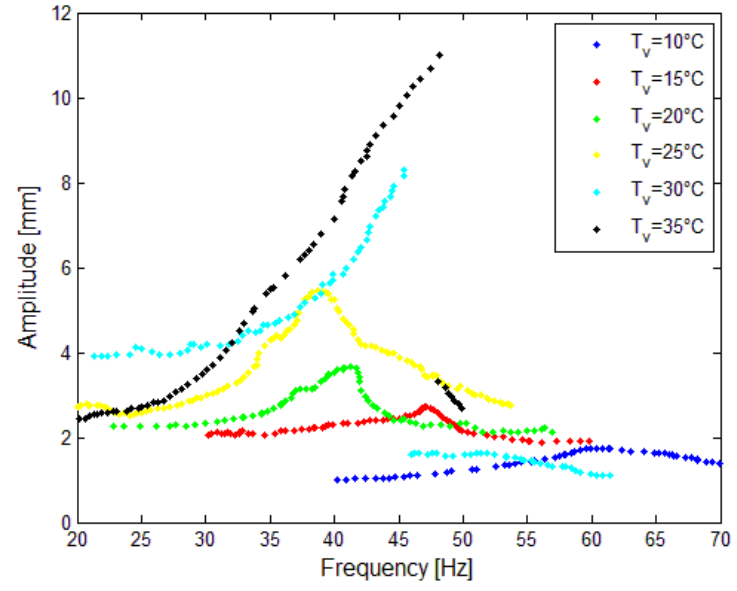

(a)

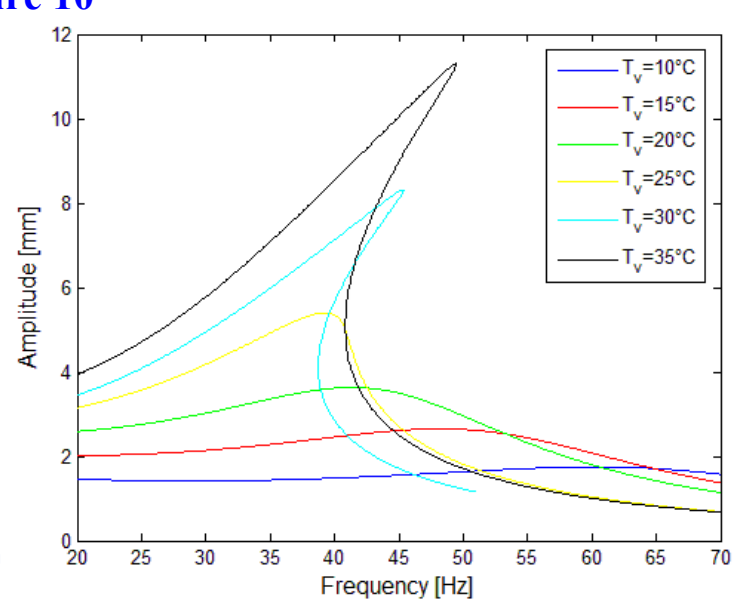

(b)

Figure 11

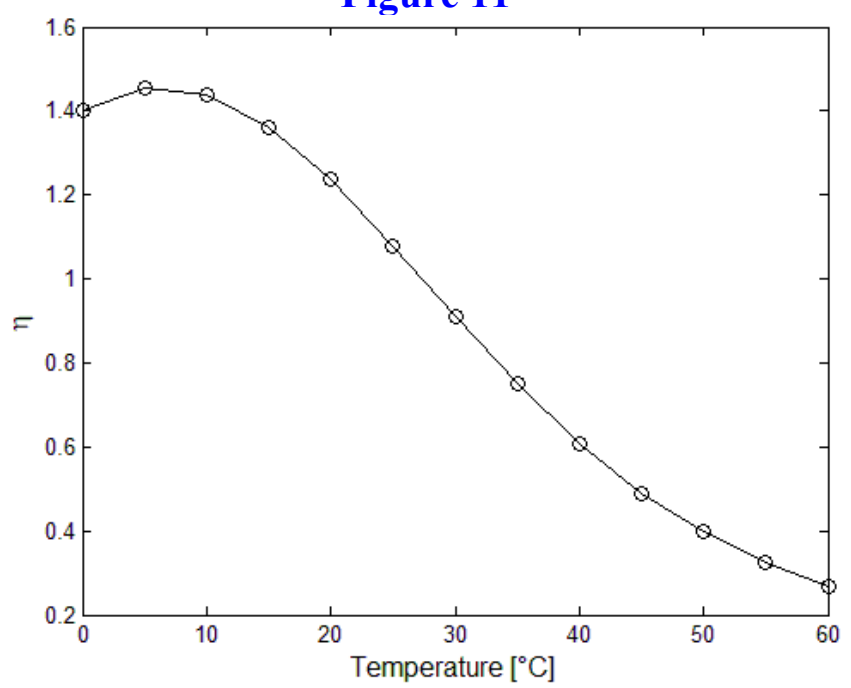


Figure 12
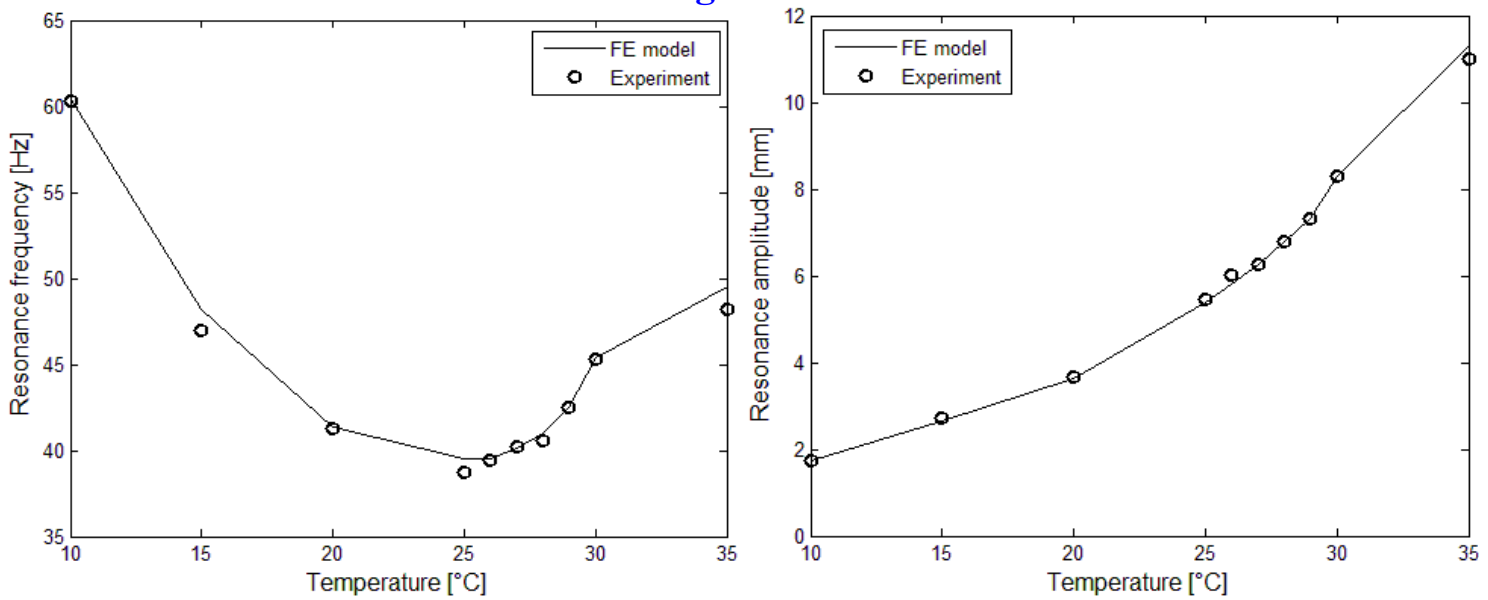

Table 1

\begin{tabular}{cccccc}
\hline \multirow{2}{*}{ Variables } & Nominal & \multicolumn{4}{c}{ Uncertainty level } \\
\cline { 3 - 6 } & Values & Case 1 & Case 2 & Case 3 & Case 4 \\
\hline$h_{e}$ & $1 \mathrm{~mm}$ & $5 \% ; 15 \%$ & --- & --- & $5 \% ; 15 \%$ \\
$h_{v}$ & $1 \mathrm{~mm}$ & --- & $5 \% ; 15 \%$ & --- & $5 \% ; 15 \%$ \\
$T_{v}$ & $30^{\circ} \mathrm{C}$ & --- & --- & $5 \% ; 15 \%$ & $5 \% ; 15 \%$ \\
\hline
\end{tabular}

Figure 13
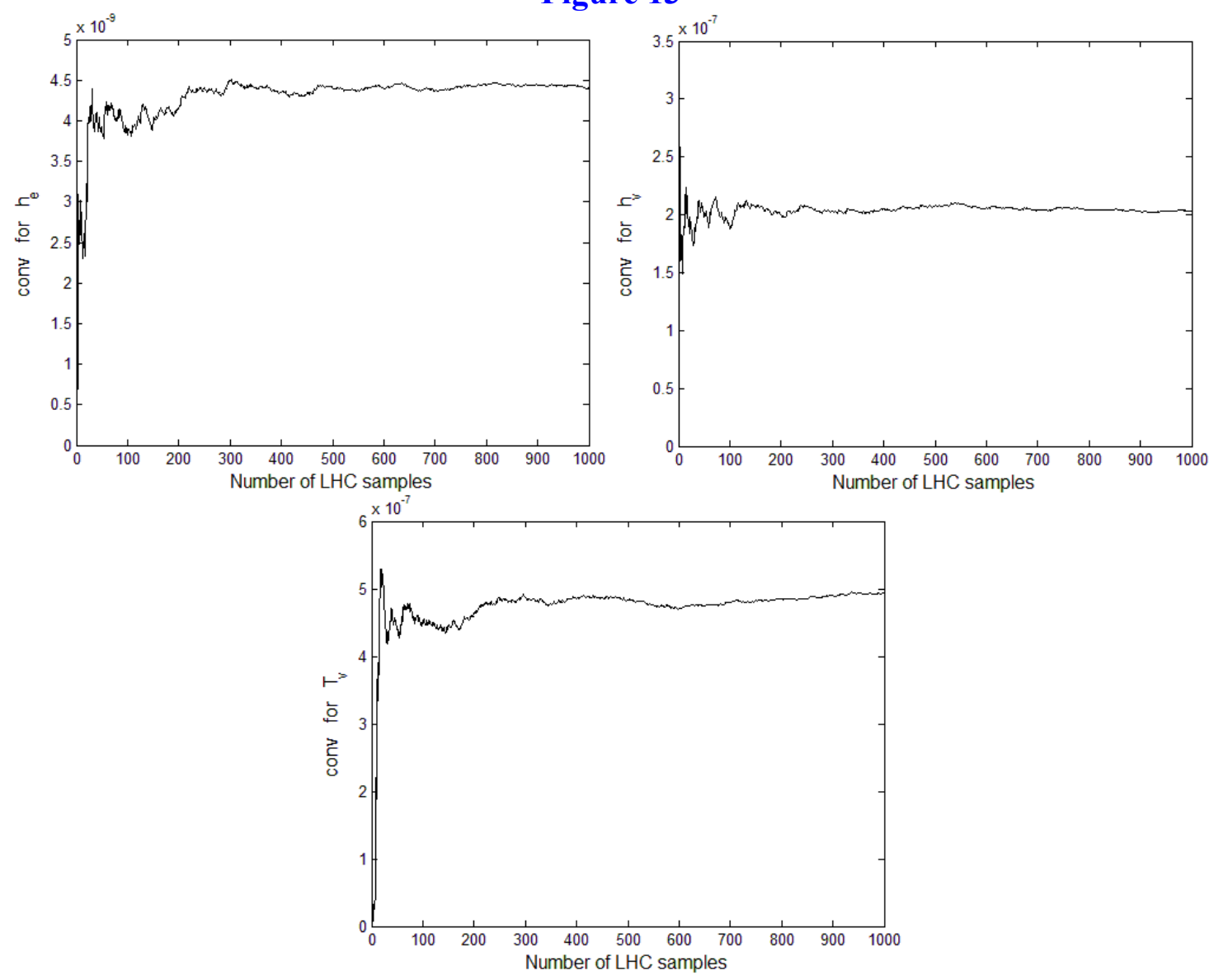
Figure 14
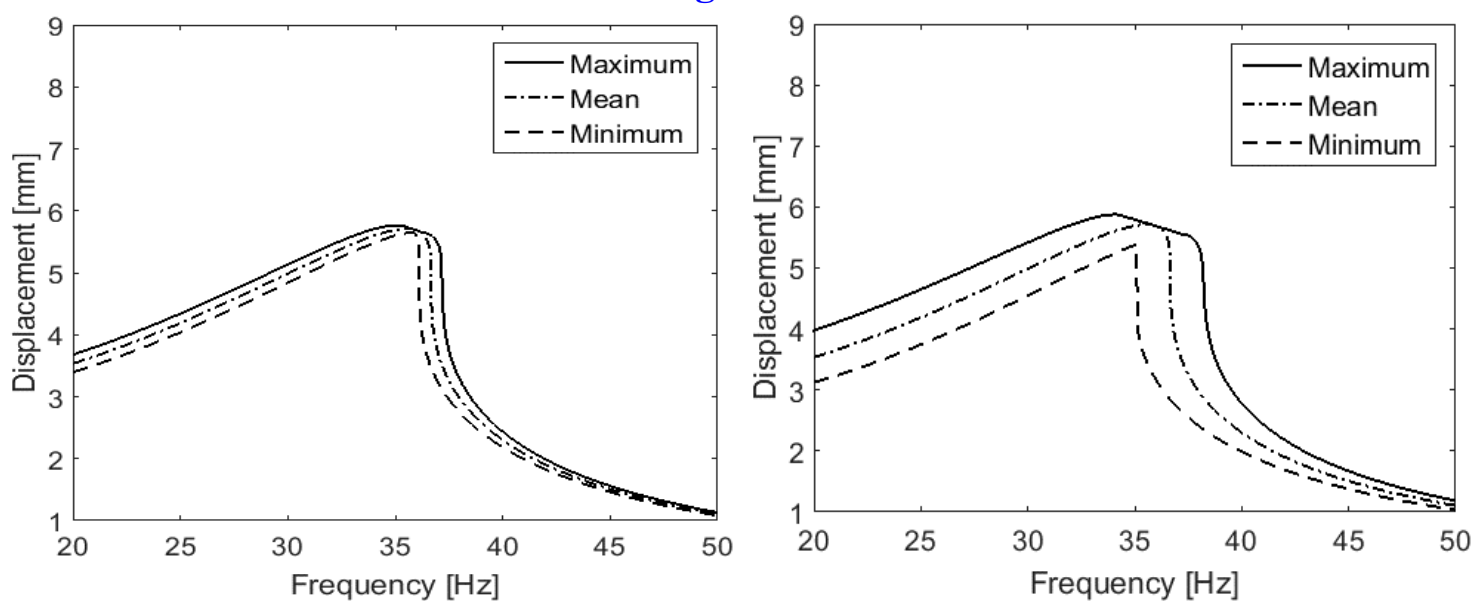

Figure 15
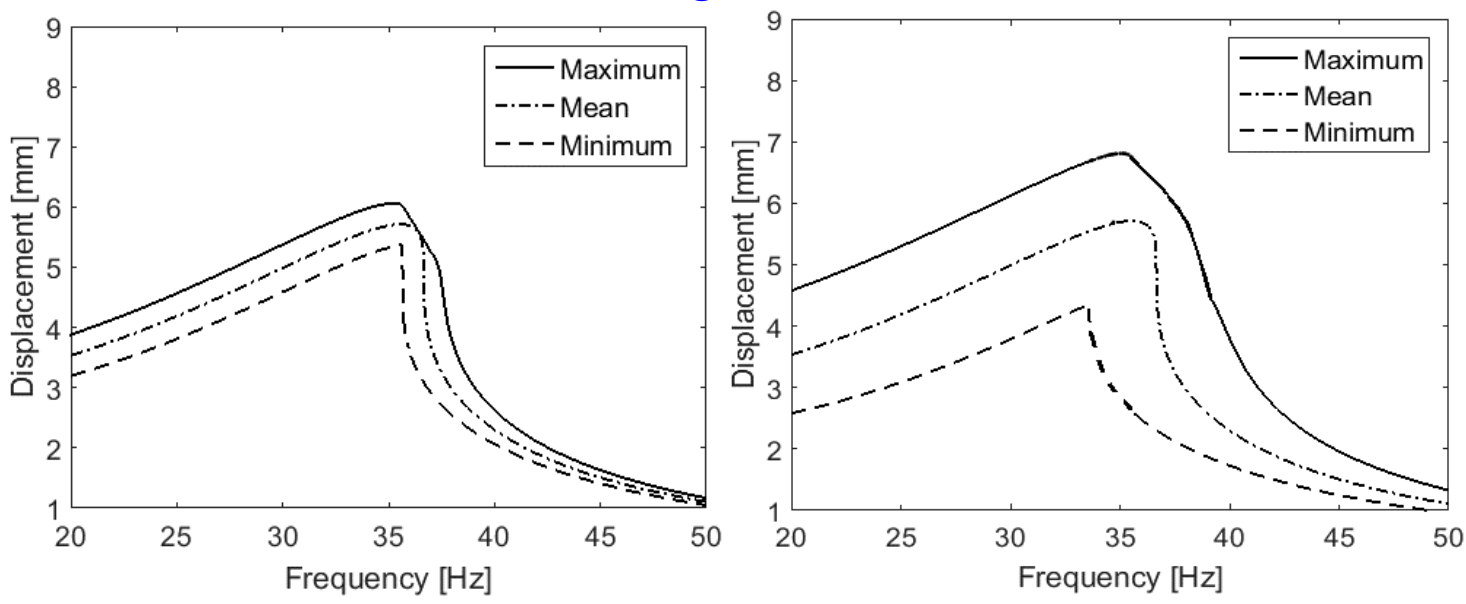

Figure 16
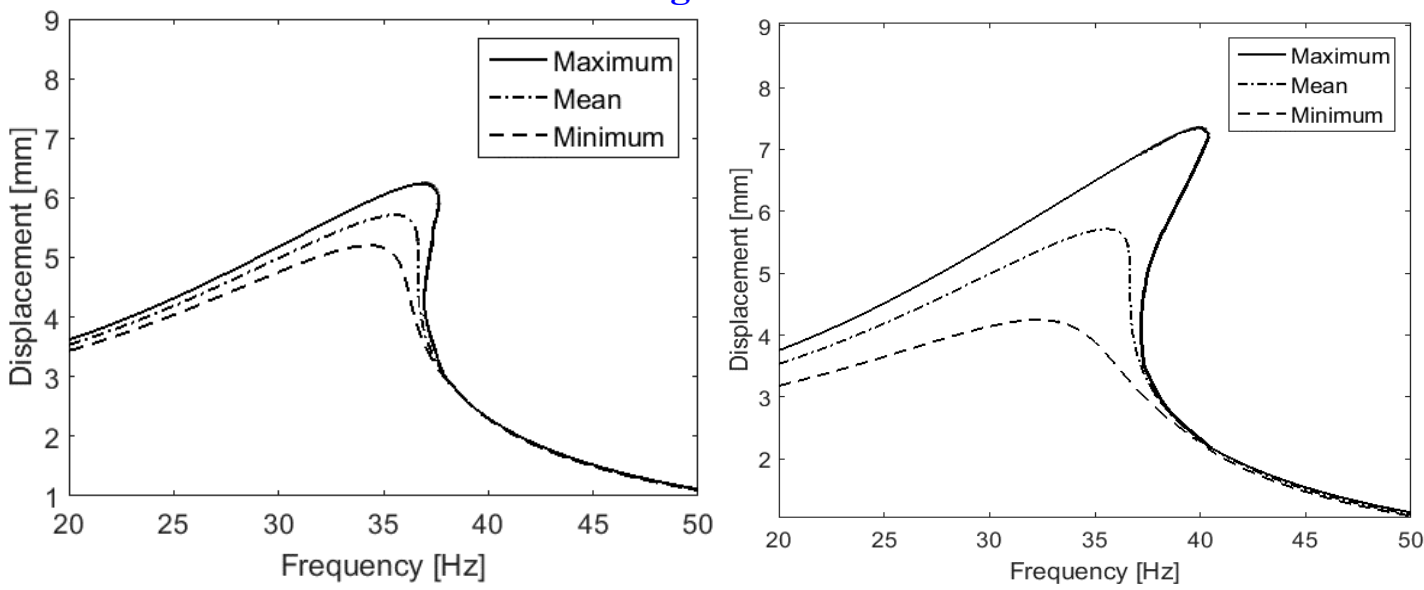
Figure 17
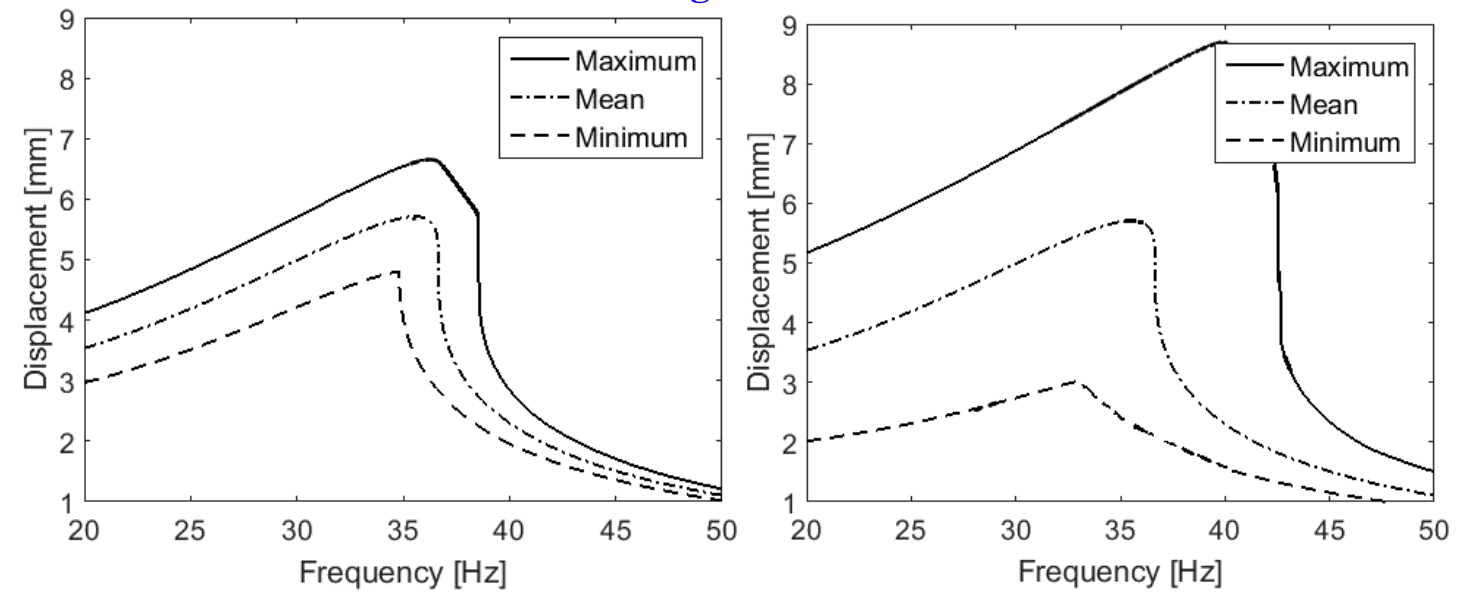